\title{
NFE2L2 Is a Potential Prognostic Biomarker and Is Correlated with Immune Infiltration in Brain Lower Grade Glioma: A Pan-Cancer Analysis
}

\author{
Qiang Ju $\mathbb{D},{ }^{1}$ Xinmei Li, ${ }^{2}$ Heng Zhang, ${ }^{2}$ Songxia Yan, ${ }^{1}$ Ying $\mathrm{Li}^{1},{ }^{1}$ and Yanjie Zhao $\mathbb{D}^{2}$ \\ ${ }^{1}$ Department of Blood Transfusion, The Affiliated Hospital of Qingdao University, Qingdao University, Qingdao, China \\ ${ }^{2}$ School of Public Health, Qingdao University, Qingdao, China \\ Correspondence should be addressed to Yanjie Zhao; zhaoyj@qdu.edu.cn
}

Received 18 June 2020; Revised 17 August 2020; Accepted 20 September 2020; Published 10 October 2020

Academic Editor: Bin Duan

Copyright ( 2020 Qiang Ju et al. This is an open access article distributed under the Creative Commons Attribution License, which permits unrestricted use, distribution, and reproduction in any medium, provided the original work is properly cited.

\begin{abstract}
Nuclear factor, erythroid 2 like 2 (NFE2L2, NRF2) is a transcription factor that regulates various antioxidant enzymes. It plays a vital physiological role in regulating oxidative stress and inflammatory response. However, the roles of NFE2L2 in human cancers are still unclear. Our study is aimed at analyzing the prognostic value of NFE2L2 in pan-cancer and at revealing the relationship between NFE2L2 expression and tumor immunity. The present study revealed that NFE2L2 was abnormally expressed and significantly correlated with mismatch repair (MMR) gene mutation levels and DNA methyltransferase expression in human pan-cancer. In particular, pan-cancer survival analysis indicated that NFE2L2 expression was associated with adverse outcomes-overall survival (OS), disease-specific survival (DSS), and progression-free interval (PFI)-in adrenocortical carcinoma (ACC), brain lower grade glioma (LGG), and pancreatic adenocarcinoma (PAAD) patients. A positive relationship was also found between NFE2L2 expression and immune infiltration, including B cells, CD4+ T cells, CD8+ T cells, neutrophils, macrophages, and dendritic cells, especially in breast invasive carcinoma (BRCA), colon adenocarcinoma (COAD), kidney renal clear cell carcinoma (KIRC), LGG, liver hepatocellular carcinoma (LIHC), and prostate adenocarcinoma (PRAD). Additionally, NFE2L2 expression was positively correlated with the immune score and the expression of immune checkpoint markers in LGG. In conclusion, these results indicate that transcription factor NFE2L2 is a potential prognostic biomarker and is correlated with immune infiltration in LGG.
\end{abstract}

\section{Introduction}

Nuclear factor, erythroid 2 like 2 (NFE2L2) is a redoxsensitive transcription factor localized mainly in the cytoplasm. It is ubiquitously expressed in the esophagus, thyroid, and other tissues $[1,2]$. NFE2L2-mediated oxidative stress is a prominent feature of cervical cancer [3], promoting the proliferation, inhibiting the apoptosis, and enhancing the migration and invasion of cervical cancer cells $[4,5]$, as well as increasing the tumor chemoresistance [6, 7], suggesting that NFE2L2 may be a marker of poor prognosis in cervical cancer patients [8]. In addition, the redox subtype of lung squamous cell carcinoma (LUSC) is driven by genomic mutations in the NFE2L2/KEAP1 complex [9]. Although cervical cancer and LUSC have been studied, many research gaps remain across the cancer spectrum.

The tumor microenvironment (TME) is very complex and contains both the cellular and noncellular components. On the one hand, inflammatory cells, including neutrophils and myeloid-derived suppressor cells (MDSCs), suppress beneficial immune functions in the TME, preventing normal immune cells from attacking tumor cells and promoting tumor growth $[10,11]$. On the other hand, immune cell infiltration of the TME constitutes a strategy used by tumor cells to evade immune-mediated killing [12-14]. Tumorassociated macrophages (TAMs) mediate immune escape and then play important roles in tumorigenesis and development [15-18]. Currently, immunotherapy is a trending topic 
in tumor therapy. For example, programmed death-1 (PD-1) and programmed death-ligand-1 (PD-L1) inhibitors have been found to induce tumor cell apoptosis by blocking the PD-1/PD-L1 signaling pathway, thus playing an effective antitumor role in lung cancer and melanoma [13, 19-21]. However, the role and underlying mechanisms of NFE2L2 in tumor immunity are unknown.

In the current study, we comprehensively analyzed the association between NFE2L2 expression and patients' prognosis in 33 cancer types. In addition, we explored the correlation between NFE2L2 expression and tumor immunity. Our findings revealed the possible role of NFE2L2 across cancers, suggesting that NFE2L2 is a potential prognostic biomarker and is correlated with immune infiltration in many cancers, especially in LGG.

\section{Materials and Methods}

2.1. Sample Information and NFE2L2 Expression Analysis in Human Pan-Cancer. NFE2L2 gene expression data in 31 normal tissues and 21 tumor cell lines were obtained from the Genotype-Tissue Expression (GTEx) portal (https:// gtexport.org/home/) and Cancer Cell Line Encyclopedia (CCLE) database (https://portals.broadinstitute.org/ccle/ about). The difference in NFE2L2 expression between cancer and normal tissues was analyzed by combining the data for normal tissues from the GTEx database with the data from The Cancer Genome Atlas (TCGA). Level 3 RNA sequencing data and clinical follow-up information for patients with 33 types of cancers (ACC: adrenocortical carcinoma; BLCA: bladder urothelial carcinoma; BRCA: breast invasive carcinoma; CESC: cervical squamous cell carcinoma; CHOL: cholangiocarcinoma; COAD: colon adenocarcinoma; DLBC: lymphoid neoplasm diffuse large B cell lymphoma; ESCA: esophageal carcinoma; GBM: glioblastoma multiforme; LGG: brain lower grade glioma; HNSC: head and neck squamous cell carcinoma; KICH: kidney chromophobe; KIRC: kidney renal clear cell carcinoma; KIRP: kidney renal papillary cell carcinoma; LAML: acute myeloid leukemia; LIHC: liver hepatocellular carcinoma; LUAD: lung adenocarcinoma; LUSC: lung squamous cell carcinoma; MESO: mesothelioma; OV: ovarian serous cystadenocarcinoma; PAAD: pancreatic adenocarcinoma; PCPG: pheochromocytoma and paraganglioma; PRAD: prostate adenocarcinoma; READ: rectum adenocarcinoma; SARC: sarcoma; SKCM: skin cutaneous melanoma; STAD: stomach adenocarcinoma; TGCT: testicular germ cell tumors; THCA: thyroid carcinoma; THYM: thymoma; UCEC: uterine corpus endometrial carcinoma; UCS: uterine carcinosarcoma; and UVM: uveal melanoma) were obtained from TCGA database. All expression data were normalized through $\log 2$ conversion.

2.2. MMR Gene Mutation and DNA Methyltransferase Analysis. Abnormalities in the DNA mismatch repair system (MMRs) can lead to tumorigenesis [22]. The mutation levels of 5 MMR genes (MLH1, MSH2, MSH6, PMS2, and EPCAM) were obtained from TCGA database. Pearson correlation analysis was used to evaluate the relationship between NFE2L2 expression and MMR gene mutation levels.
In addition, DNA methyltransferases play an important role in altering chromatin structure and gene expression [23]. The relationship between the expression level of NFE2L2 and that of 4 methyltransferases (DNMT1, DNMT2, DNMT3A, and DNMT3B) was evaluated by Pearson correlation analysis.

2.3. Survival and Prognosis Analysis. The relationship between NFE2L2 gene expression and patients' prognosis (OS: overall survival; DSS: disease-specific survival; DFI: disease-free interval; and PFI: progression-free interval) in 33 cancers was visualized with forest plots and KaplanMeier curves. The hazard ratio (HR) and 95\% confidence intervals were calculated via univariate survival analysis.

2.4. Correlations between NFE2L2 Expression and Immune Characteristics in the TIMER Database. The Tumor Immune Estimation Resource (TIMER) database contains 10,897 samples from TCGA (https://cistrome.shinyapps.io/timer/). RNA-seq expression profile data were used to evaluate the infiltration of 6 immune cells (B cells, CD4+ T cells, CD8+ $\mathrm{T}$ cells, neutrophils, macrophages, and dendritic cells) in tumor tissues. The scores of these 6 infiltrating immune cells in 33 cancers were downloaded from the TIMER database. Spearman correlation analysis was used to evaluate the correlation between NFE2L2 expression and immune infiltration. In addition, we evaluated the relationship between NFE2L2 expression and the immune/stromal scores (ImmuneScore and StromalScore) and immune checkpoint marker expression levels by Spearman and Pearson correlation analyses, respectively. Gene expression levels are shown as $\log 2$ RSEM values.

2.5. Statistical Analysis. The Kruskal-Wallis test was adopted to analyze NFE2L2 expression levels in different tissues and cancer cell lines. Differences in NFE2L2 expression levels in tumor tissues and normal tissues were evaluated by a $t$-test. In survival analysis, the HRs and $P$ value were calculated by univariate Cox regression analysis. KaplanMeier curves were used to compare the survival of patients stratified according to different levels of NFE2L2 expression. $P<0.05$ was set as the significance threshold for all statistical analyses.

\section{Results}

3.1. The mRNA Expression Level of NFE2L2 in Human PanCancer. First, we analyzed NFE2L2 expression in 31 types of tissues using the GTEx dataset. As shown in Figure 1(a), NFE2L2 was generally highly expressed in the bladder, ovary, vagina, and thyroid tissues. Furthermore, we downloaded the data of tumor cell lines from the CCLE database and analyzed NFE2L2 expression in these tumor cell lines. Results showed that NFE2L2 was expressed in all 21 kinds of tumor cell lines (Figure 1(b)). To further determine the differences in NFE2L2 expression between the tumor and normal tissues, we obtained NFE2L2 expression data from TCGA database. As shown in Figure 1(c), NFE2L2 expression was significantly higher in CHOL and LUSC tissues than in normal tissues. However, it was significantly lower in BLCA, BRCA, COAD, KICH, KIRC, KIRP, LIHC, LUAD, PRAD, READ, 


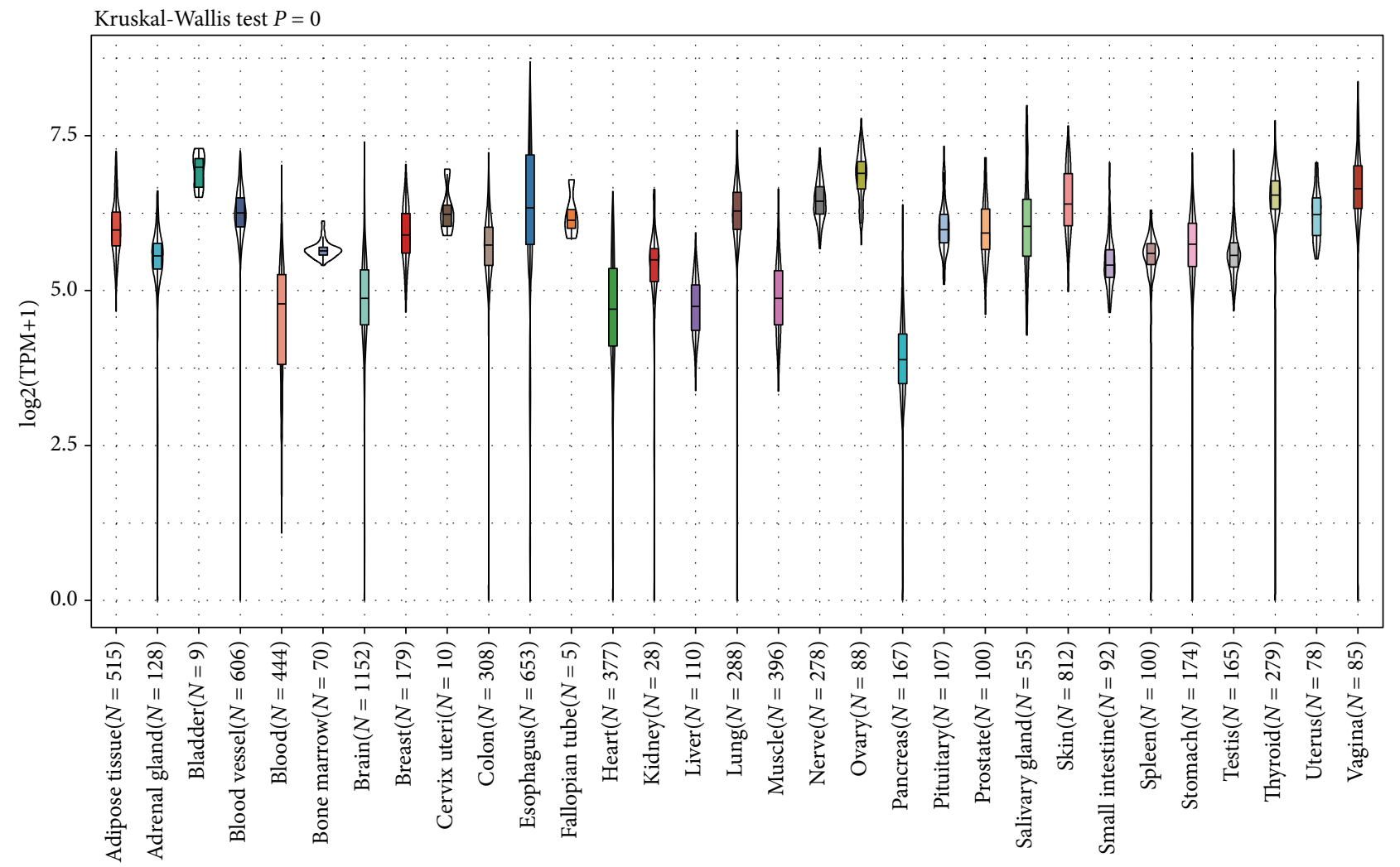

(a)

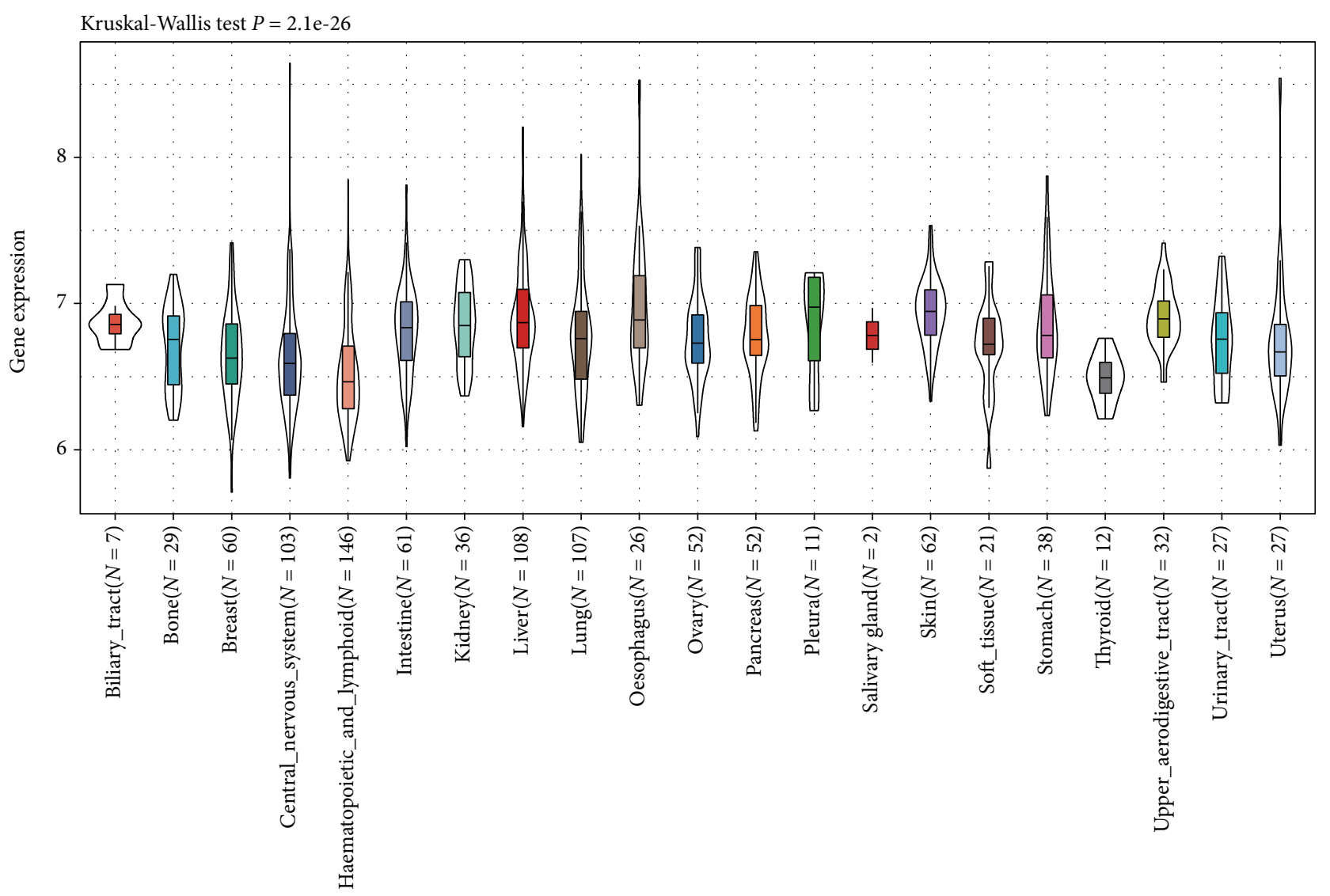

(b)

Figure 1: Continued. 


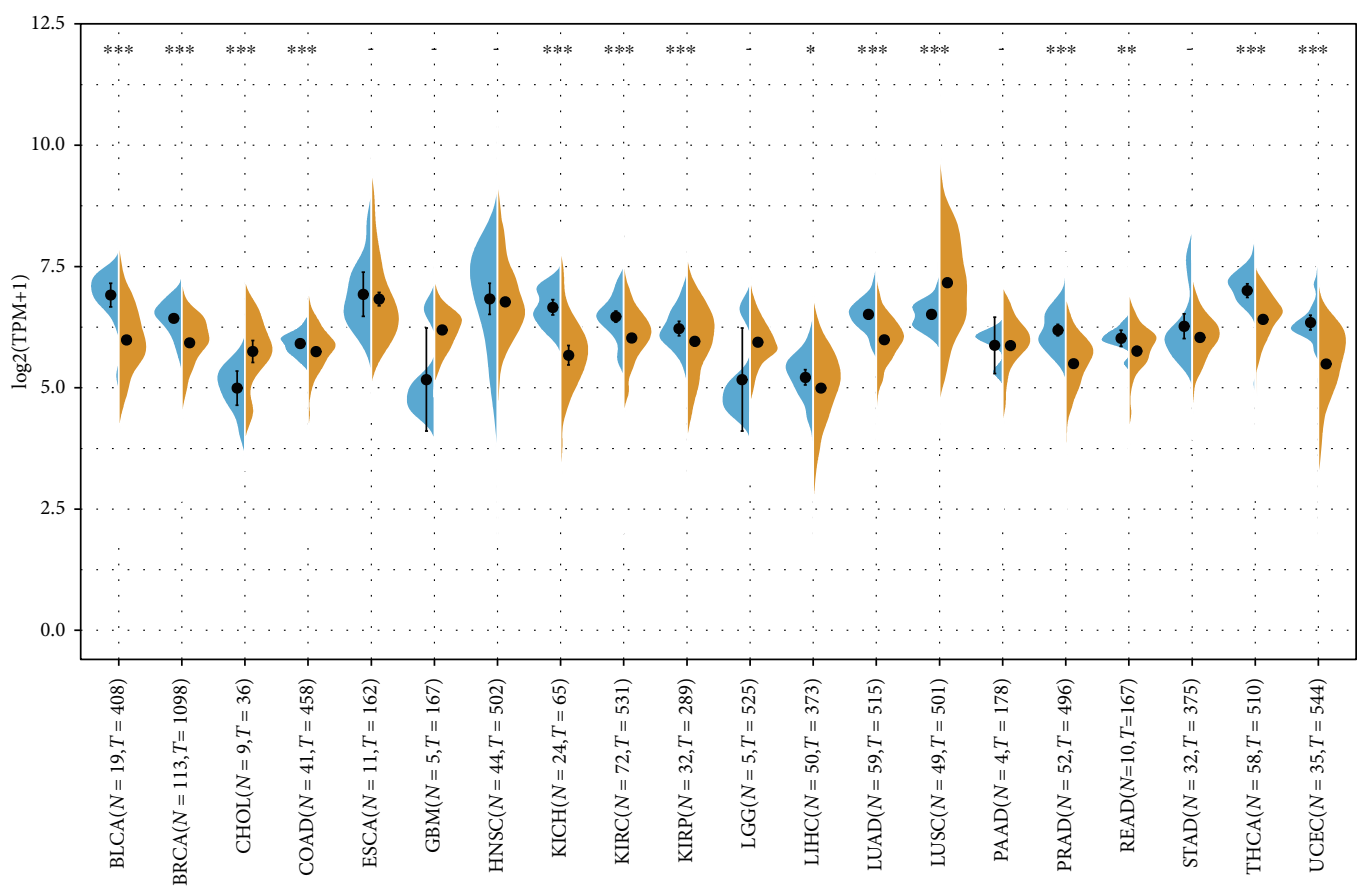

Group
- Normal
- Tumor

(c)

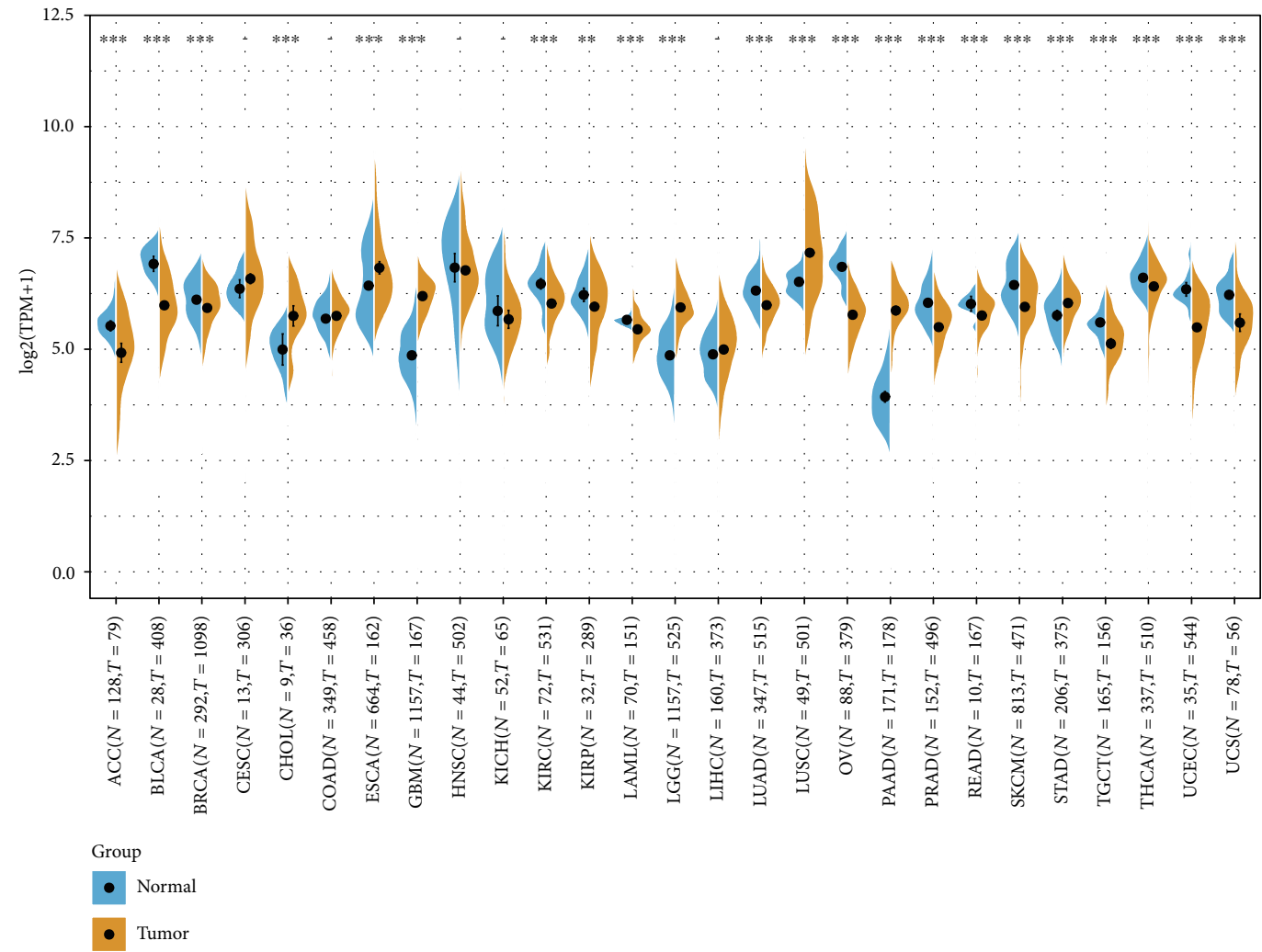

(d)

FIGURE 1: NFE2L2 is abnormally expressed in pan-cancer. (a) NFE2L2 expression in 31 normal tissues from the GTEx database. (b) NFE2L2 expression in 21 tumor cells from the CCLE database. (c) Differential expression of NFE2L2 in cancers and normal tissues from TCGA database. (d) NFE2L2 expression in 27 cancer types from the GTEx database and TCGA database $\left({ }^{*} P<0.05,{ }^{* *} P<0.01\right.$, and $\left.{ }^{* * *} P<0.001\right)$. 


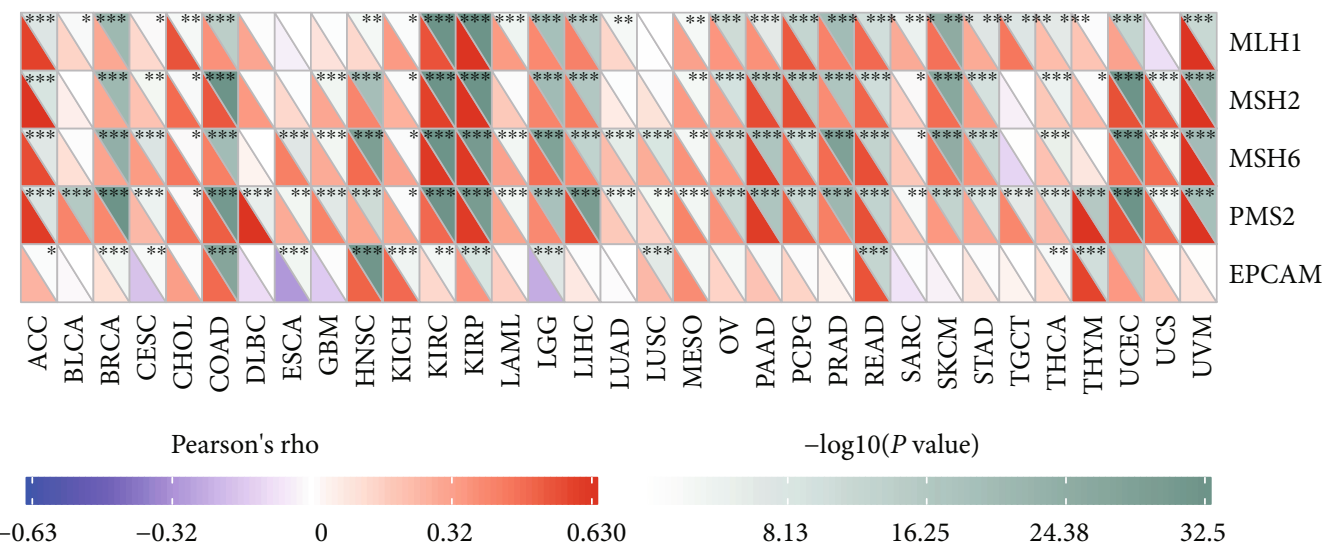

(a)

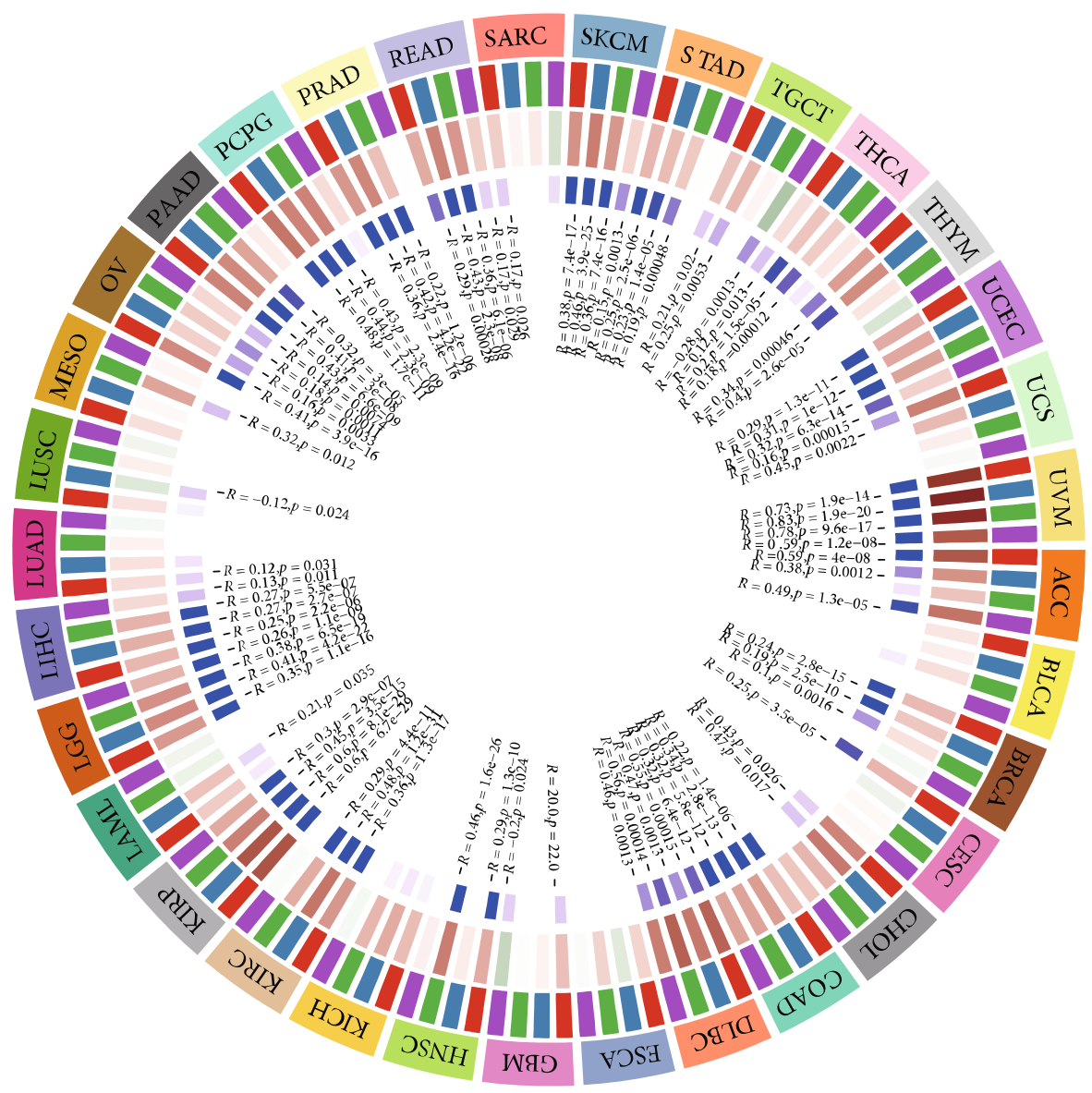

(b)

FIgURE 2: NFE2L2 expression is correlated with MMR gene mutation levels and DNA methyltransferase expression in pan-cancer. (a) Pearson correlation analysis of NFE2L2 expression with the mutation levels of 5 MMR genes (MLH1, MSH2, MSH6, PMS2, and EPCAM) in pan-cancer $\left({ }^{*} P<0.05,{ }^{* *} P<0.01\right.$, and $\left.{ }^{* * *} P<0.001\right)$. (b) Pearson correlation analysis of NFE2L2 expression with that of 4 DNA methyltransferases (DNMT1, DNMT2, DNMT3A, and DNMT3B) in pan-cancer.

THCA, and UCEC compared with normal tissues. Due to the small number of normal tissue samples in TCGA database, we further integrated the normal tissue data from the GTEx database and the tumor tissue data from TCGA database to analyze the differences in NFE2L2 expression in 27 cancer types. Results revealed that NFE2L2 was abnormally expressed in 22 of these cancers. Specifically, NFE2L2 expres- sion was higher in tissues from 7 cancers (CHOL, ESCA, GBM, LGG, LUSC, PAAD, and STAD) and lower in tissues from 15 cancers (ACC, BLCA, BRCA, KIRC, KIRP, LAML, LUAD, OV, PRAD, READ, SKCM, TGCT, THCA, UCEC, and UCS) than in the normal tissues (Figure 1(d)). Taken together, these results reveal that NFE2L2 is abnormally expressed in different cancers. 


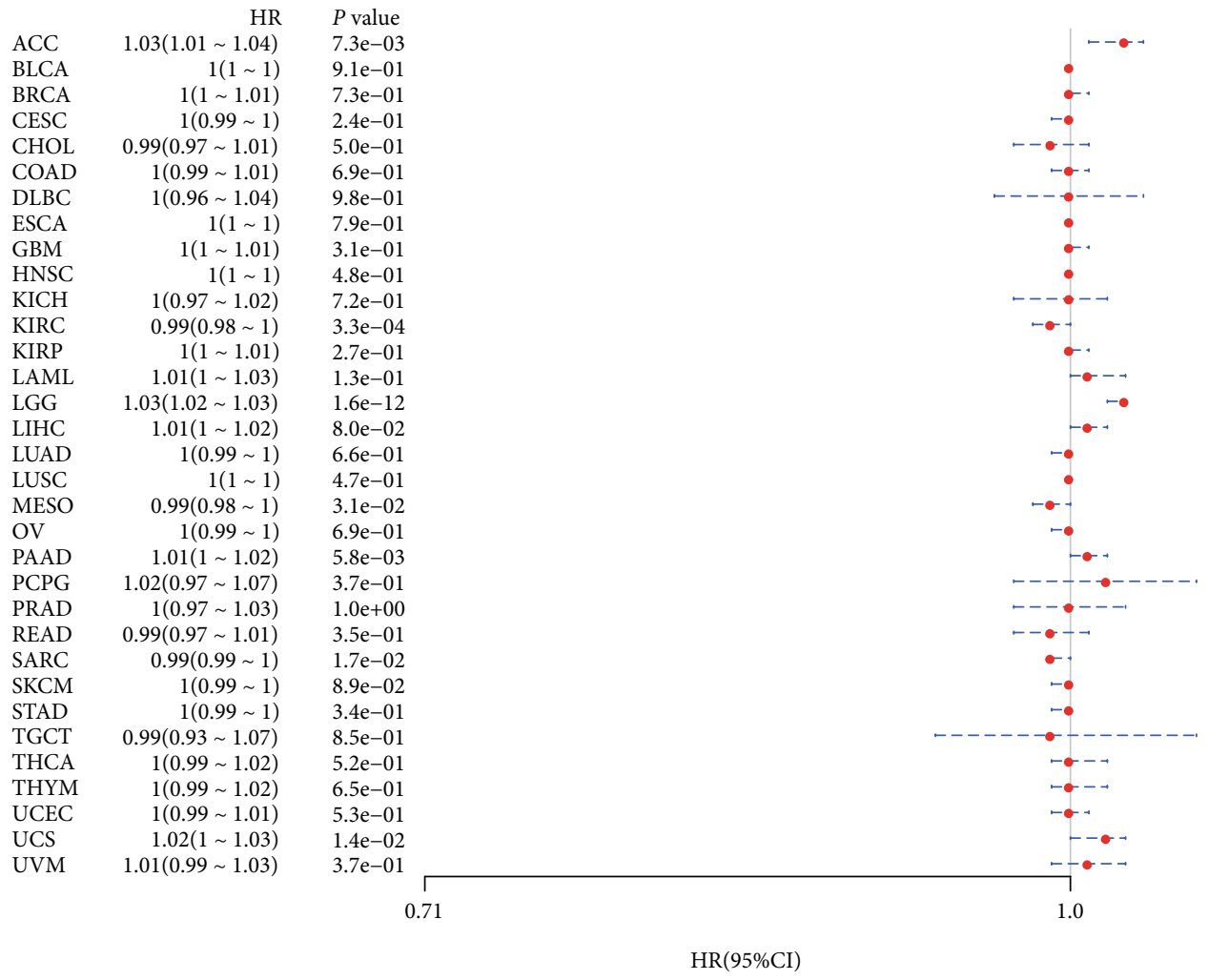

(a)

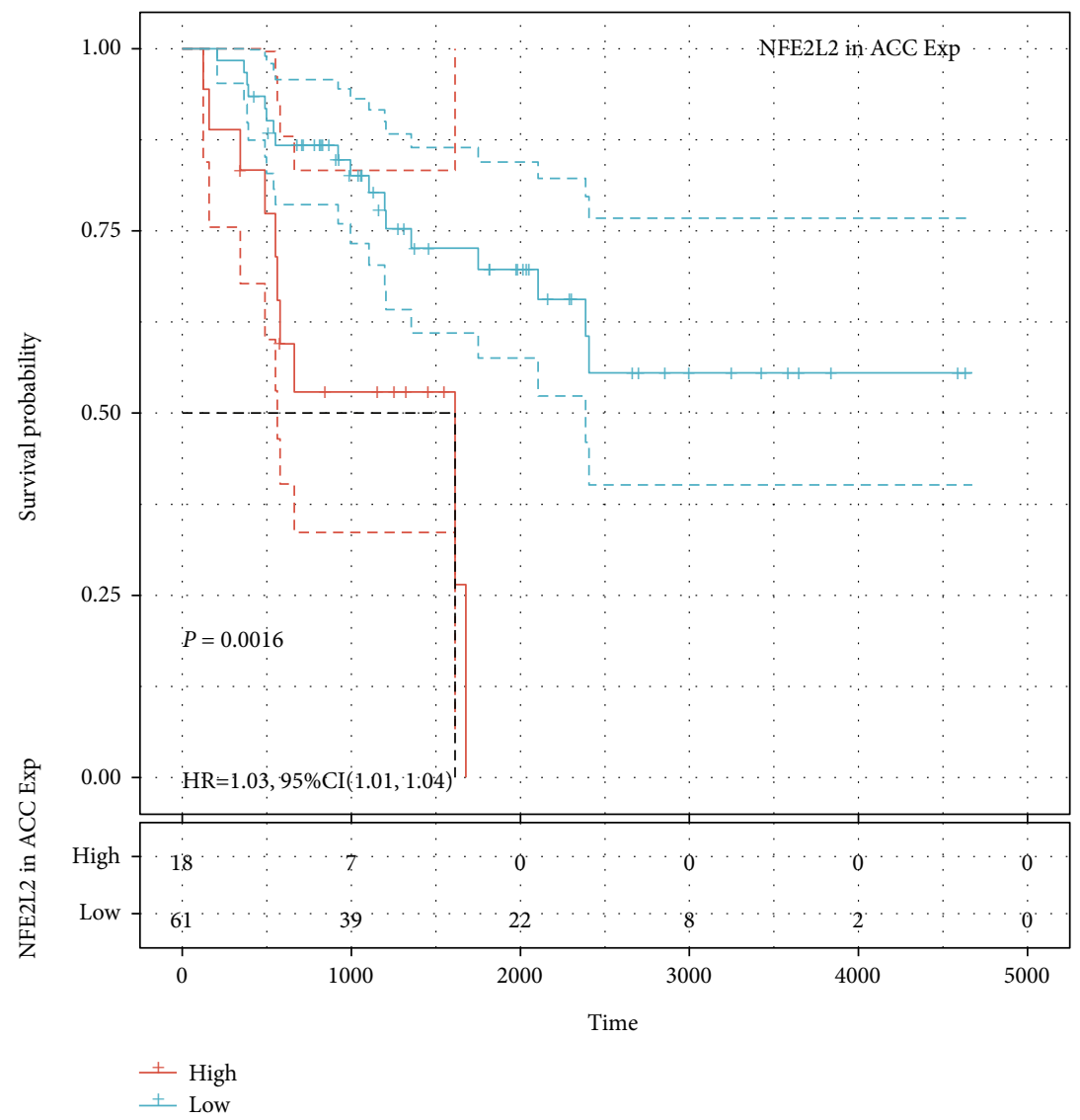

(b)

Figure 3: Continued. 


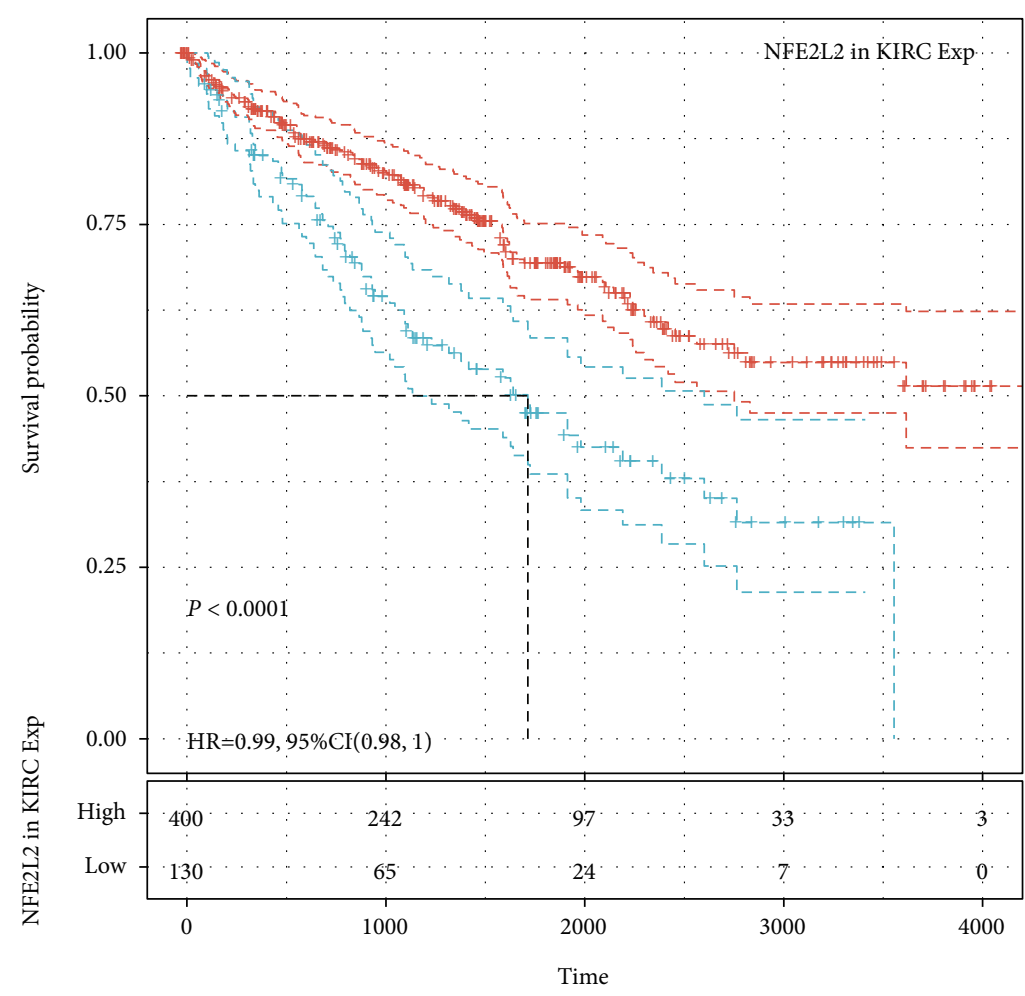

+ High

+ Low

(c)

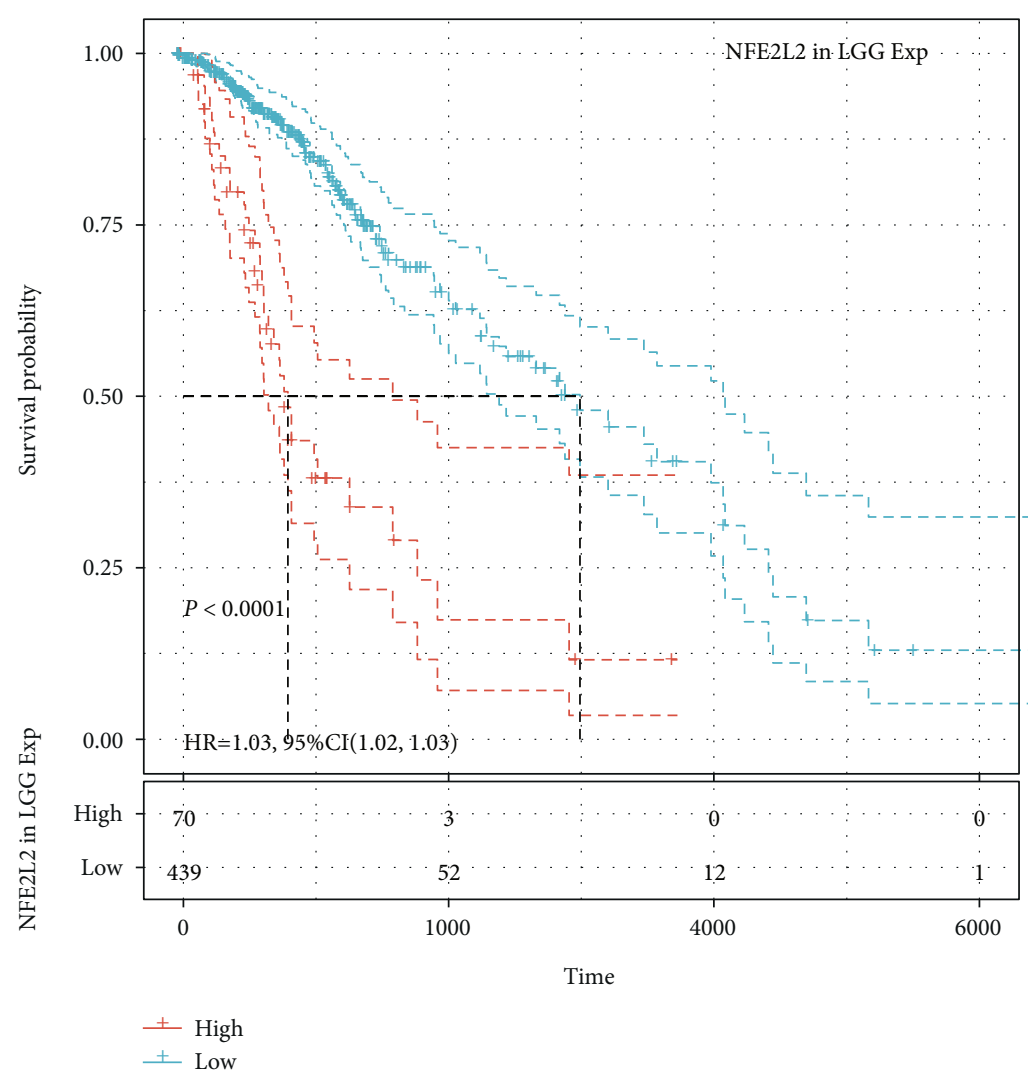

(d)

Figure 3: Continued. 


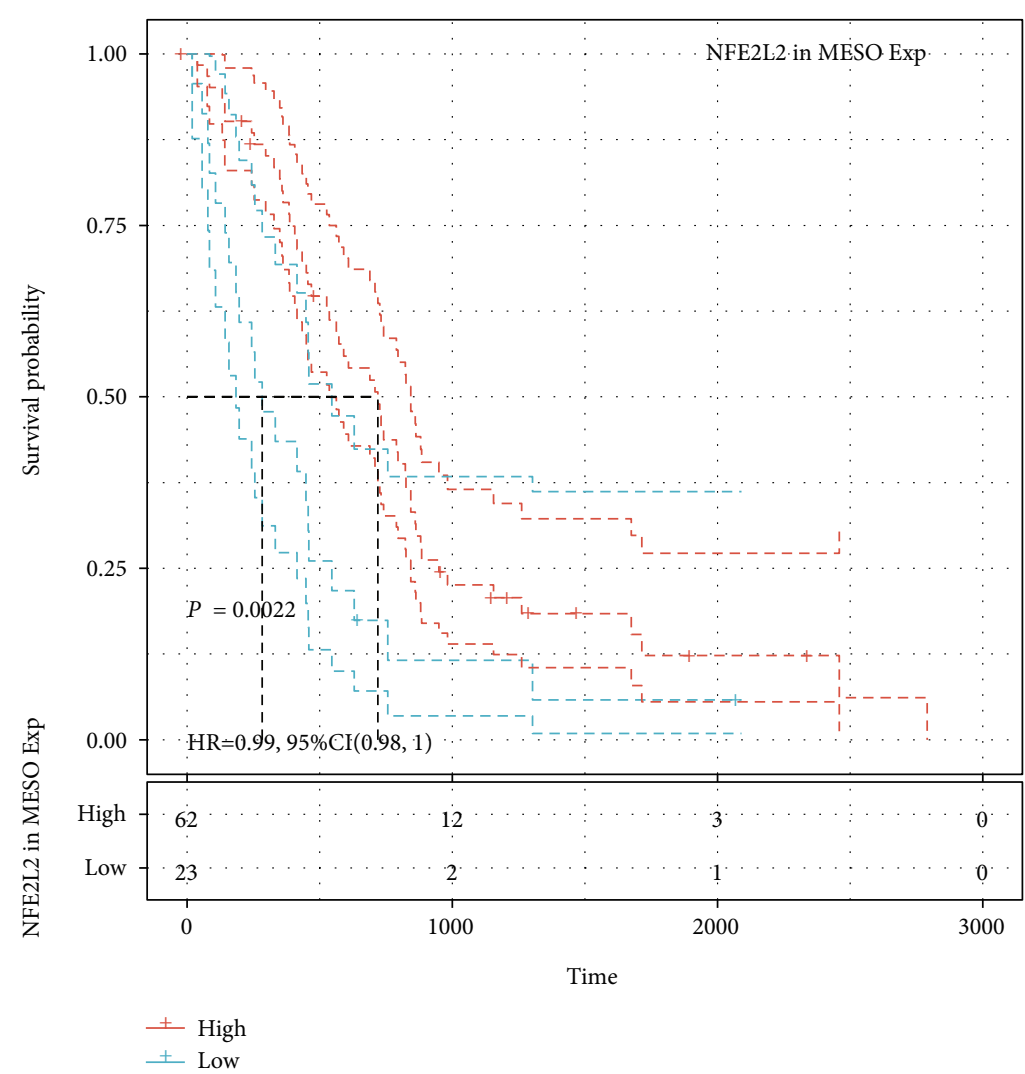

(e)

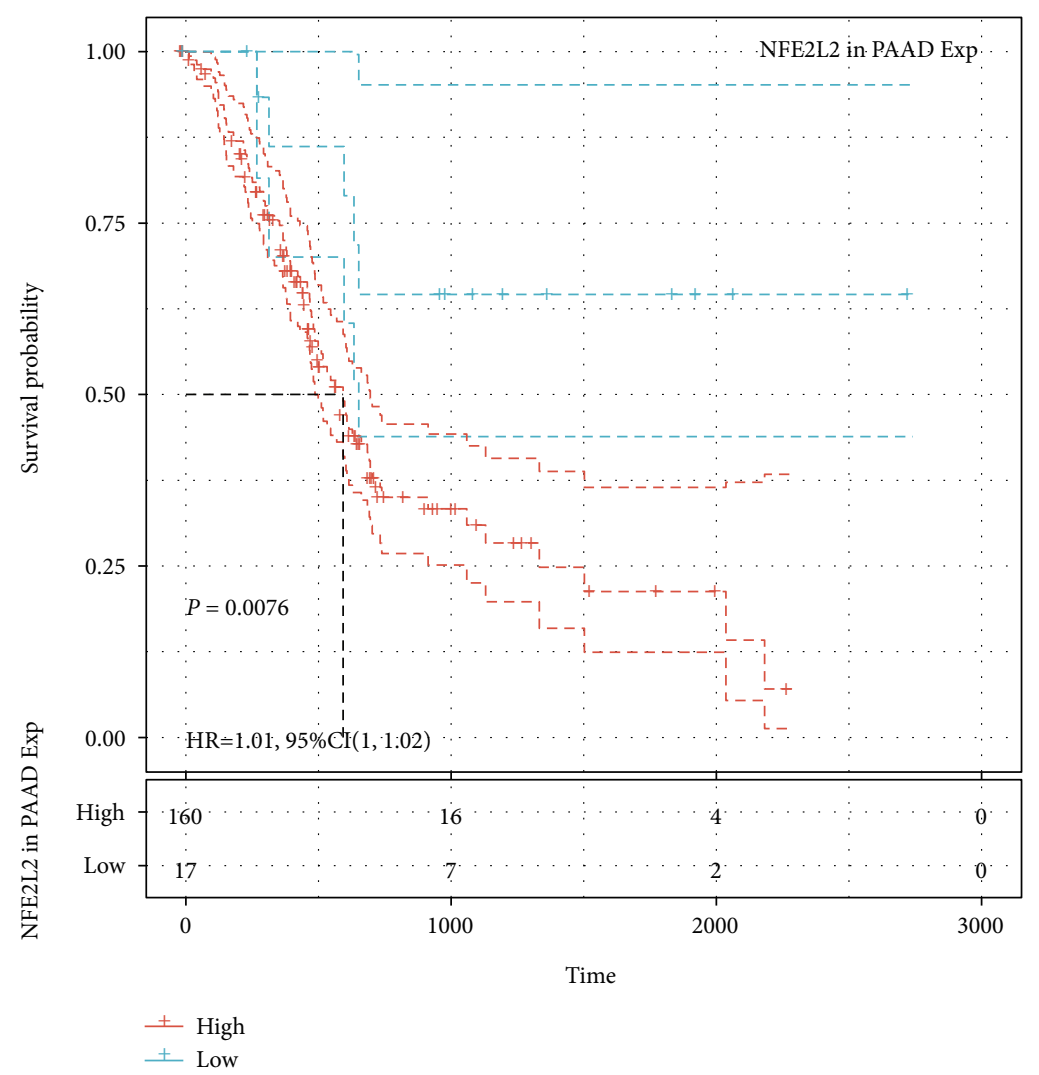

(f)

FIgURe 3: Continued. 


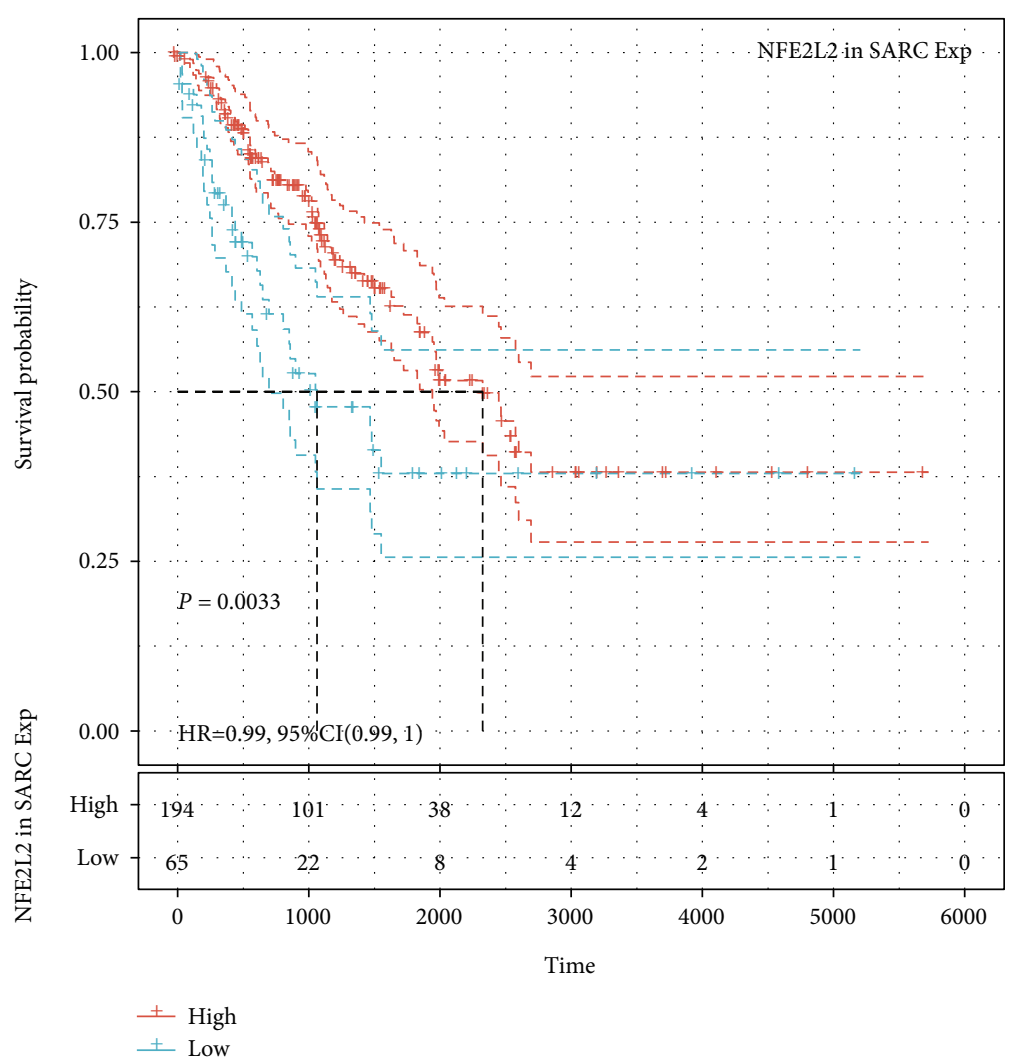

(g)

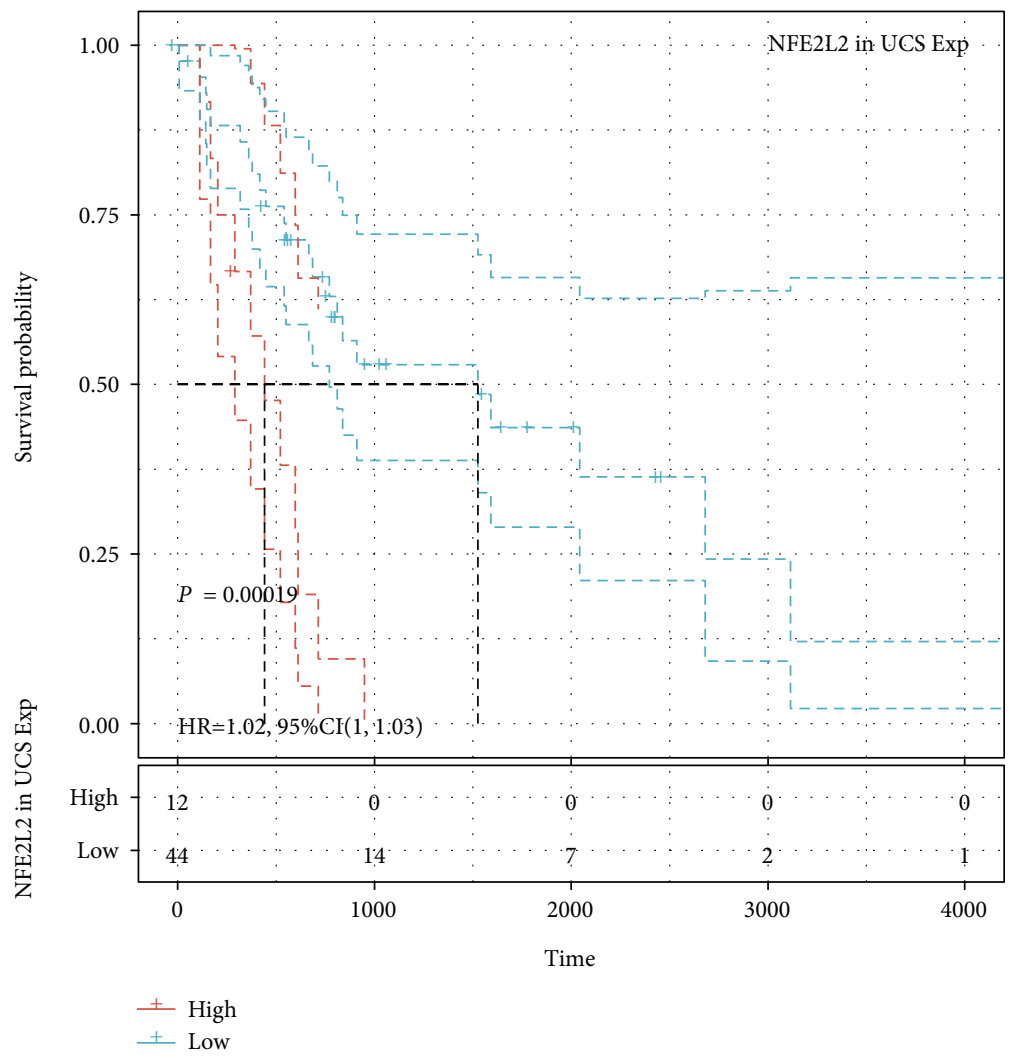

(h)

FIGURE 3: Relationship of NFE2L2 expression with patients' OS. (a) Forest plots showing the HRs related to NFE2L2 expression in 33 cancer types. (b-h) Kaplan-Meier OS curves for patients stratified by different expression levels of NFE2L2 in 7 cancer types. 


$\begin{array}{lrr} & \text { HR } & P \text { value } \\ \text { ACC } & 1.02(1 \sim 1.04) & 1.8 \mathrm{e}-02 \\ \text { BLCA } & 1(1 \sim 1) & 9.7 \mathrm{e}-01 \\ \text { BRCA } & 0.99(0.99 \sim 1) & 1.8 \mathrm{e}-01 \\ \text { CESC } & 1(0.99 \sim 1) & 2.8 \mathrm{e}-01 \\ \text { CHOL } & 0.99(0.97 \sim 1.01) & 5.7 \mathrm{e}-01 \\ \text { COAD } & 0.99(0.98 \sim 1.01) & 3.9 \mathrm{e}-01 \\ \text { DLBC } & 1(0.95 \sim 1.05) & 9.8 \mathrm{e}-01 \\ \text { ESCA } & 1(1 \sim 1) & 9.9 \mathrm{e}-01 \\ \text { GBM } & 1.01(1 \sim 1.02) & 2.5 \mathrm{e}-01 \\ \text { HNSC } & 1(1 \sim 1) & 8.8 \mathrm{e}-01 \\ \text { KICH } & 1(0.98 \sim 1.02) & 9.8 \mathrm{e}-01 \\ \text { KIRC } & 0.99(0.98 \sim 0.99) & 6.1 \mathrm{e}-05 \\ \text { KIRP } & 1.01(1 \sim 1.02) & 1.2 \mathrm{e}-01 \\ \text { LAML } & \text { NA(NA NA) } & \\ \text { LGG } & 1.03(1.02 \sim 1.04) & 1.2 \mathrm{e}-13 \\ \text { LIHC } & 1.01(0.99 \sim 1.02) & 2.6 \mathrm{e}-01 \\ \text { LUAD } & 1(0.99 \sim 1) & 8.0 \mathrm{e}-01 \\ \text { LUSC } & 1(1 \sim 1) & 1.8 \mathrm{e}-01 \\ \text { MESO } & 0.99(0.98 \sim 1) & 6.8 \mathrm{e}-02 \\ \text { OV } & 1(0.99 \sim 1) & 6.7 \mathrm{e}-01 \\ \text { PAAD } & 1.01(1 \sim 1.02) & 9.7 \mathrm{e}-03 \\ \text { PCPG } & 1.03(0.97 \sim 1.1) & 2.8 \mathrm{e}-01 \\ \text { PRAD } & 1.01(0.96 \sim 1.05) & 8.1 \mathrm{e}-01 \\ \text { READ } & 0.99(0.96 \sim 1.01) & 3.6 \mathrm{e}-01 \\ \text { SARC } & 0.99(0.99 \sim 1) & 3.3 \mathrm{e}-02 \\ \text { SKCM } & 1(0.99 \sim 1) & 1.4 \mathrm{e}-01 \\ \text { STAD } & 1(0.99 \sim 1) & 3.5 \mathrm{e}-01 \\ \text { TGCT } & 0.99(0.92 \sim 1.07) & 8.8 \mathrm{e}-01 \\ \text { THCA } & 1(0.98 \sim 1.03) & 8.6 \mathrm{e}-01 \\ \text { THYM } & 0.97(0.94 \sim 1.01) & 1.6 \mathrm{e}-01 \\ \text { UCEC } & 1.01(1 \sim 1.02) & 1.8 \mathrm{e}-01 \\ \text { UCS } & 1.01(1 \sim 1.03) & 3.8 \mathrm{e}-02 \\ \text { UVM } & 1.01(0.99 \sim 1.03) & 2.6 \mathrm{e}-01 \\ & & \end{array}$

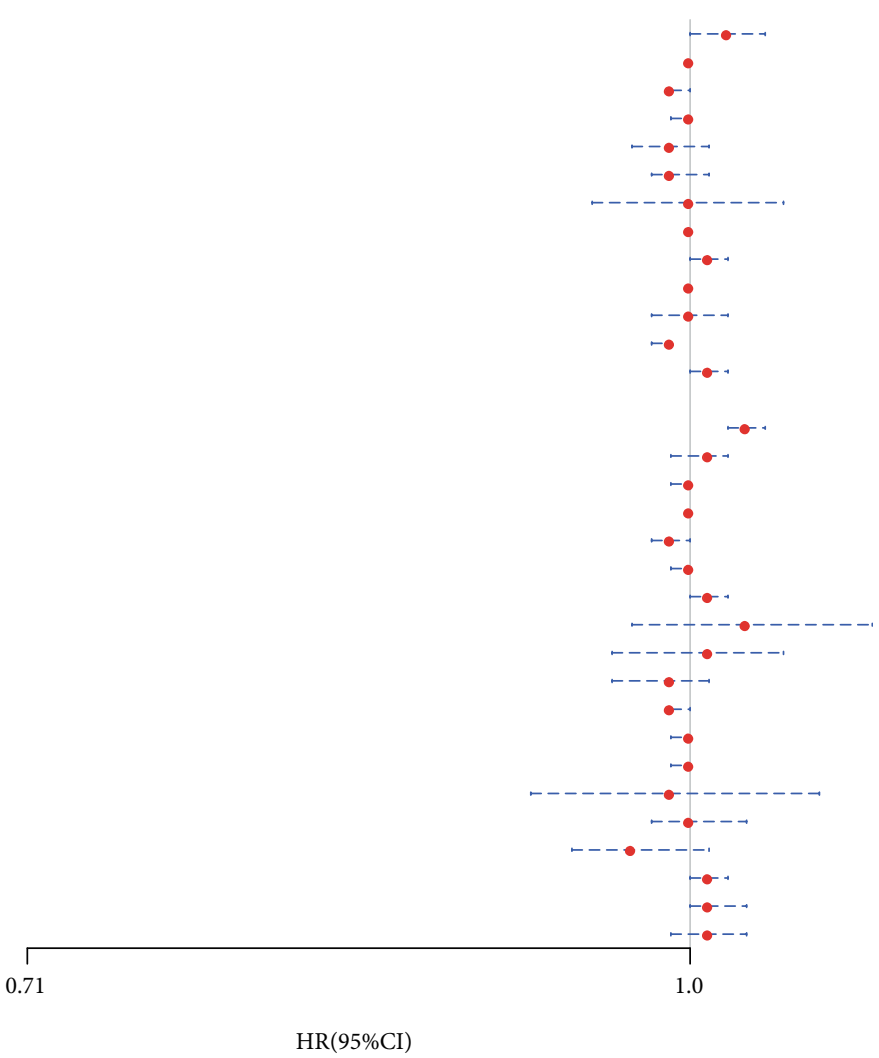

(a)

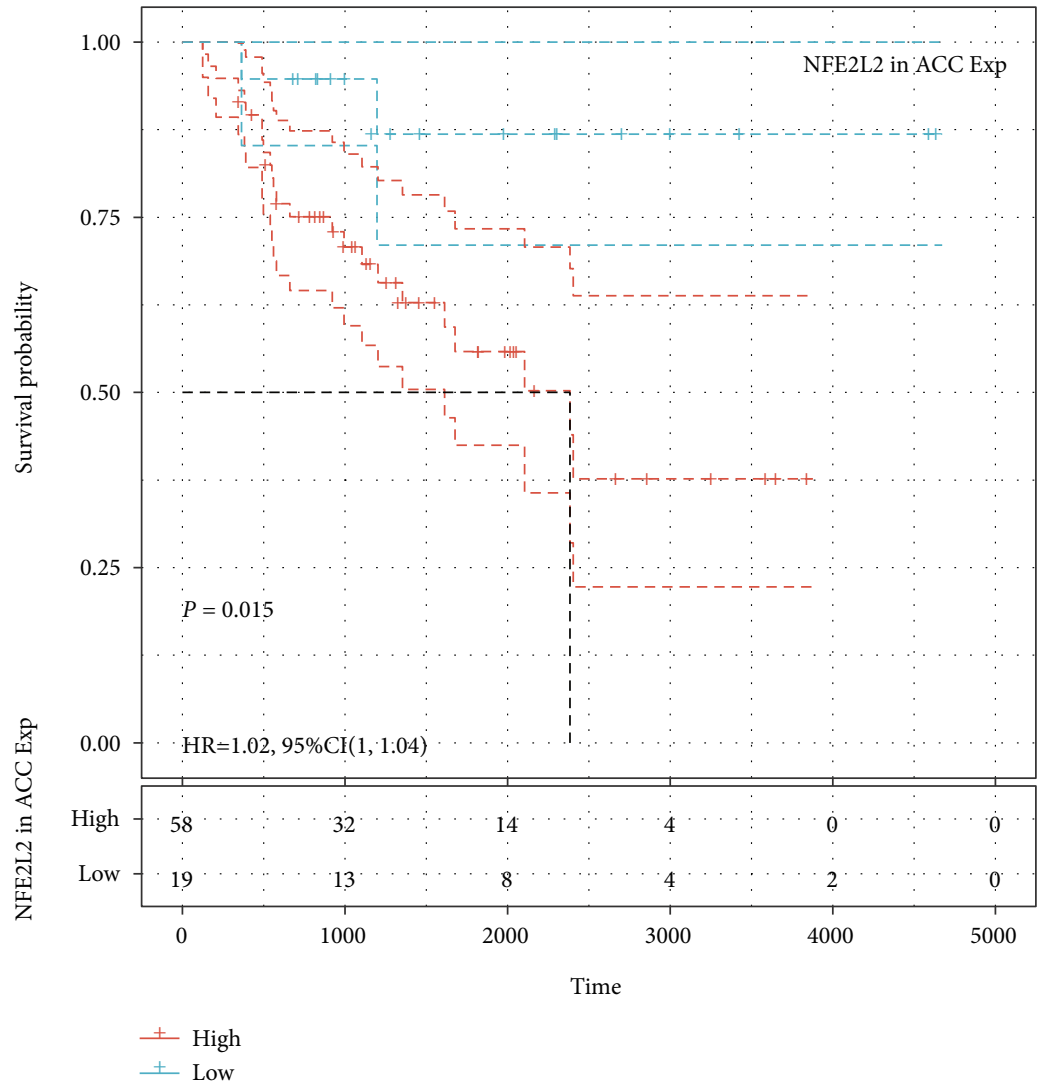

(b)

FIgURE 4: Continued. 


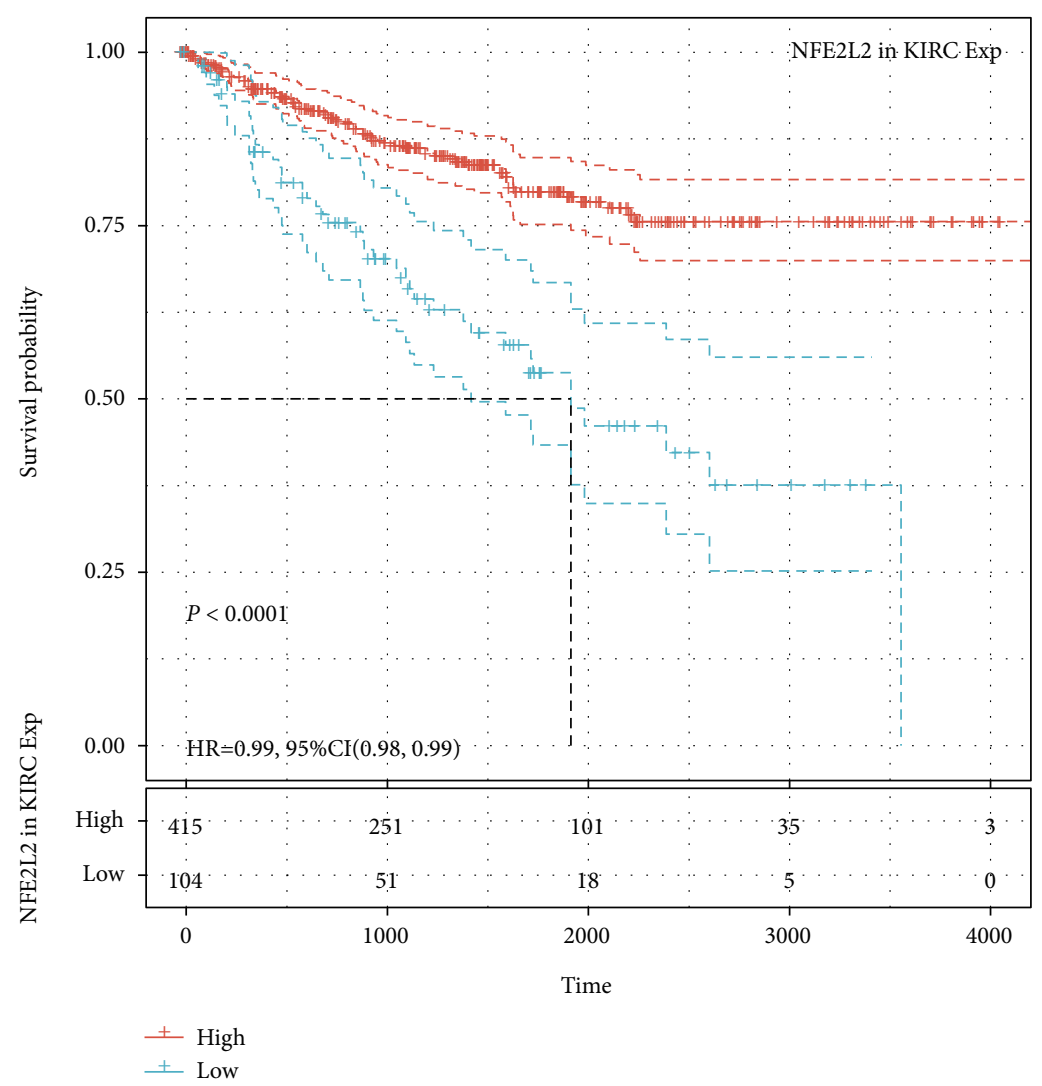

(c)

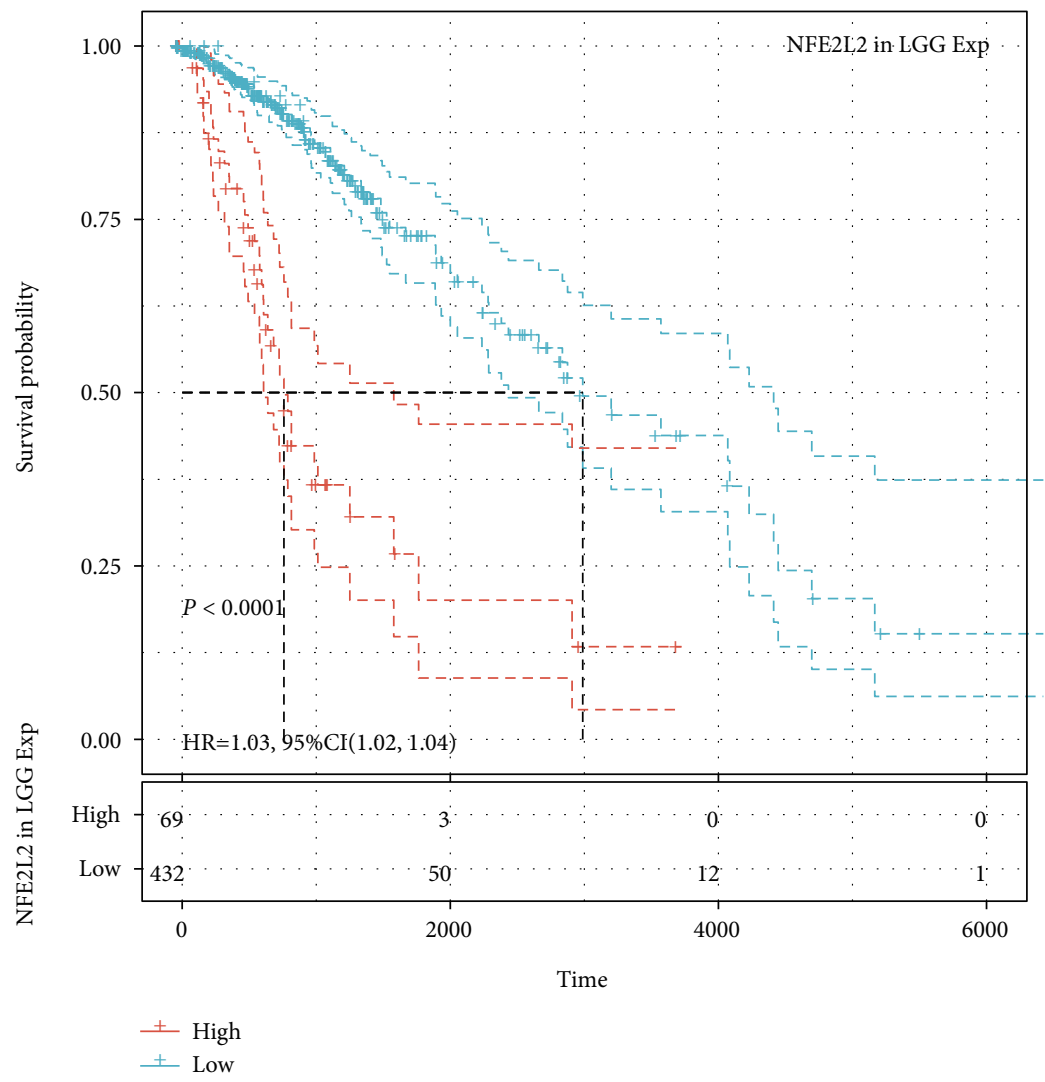

(d)

Figure 4: Continued. 


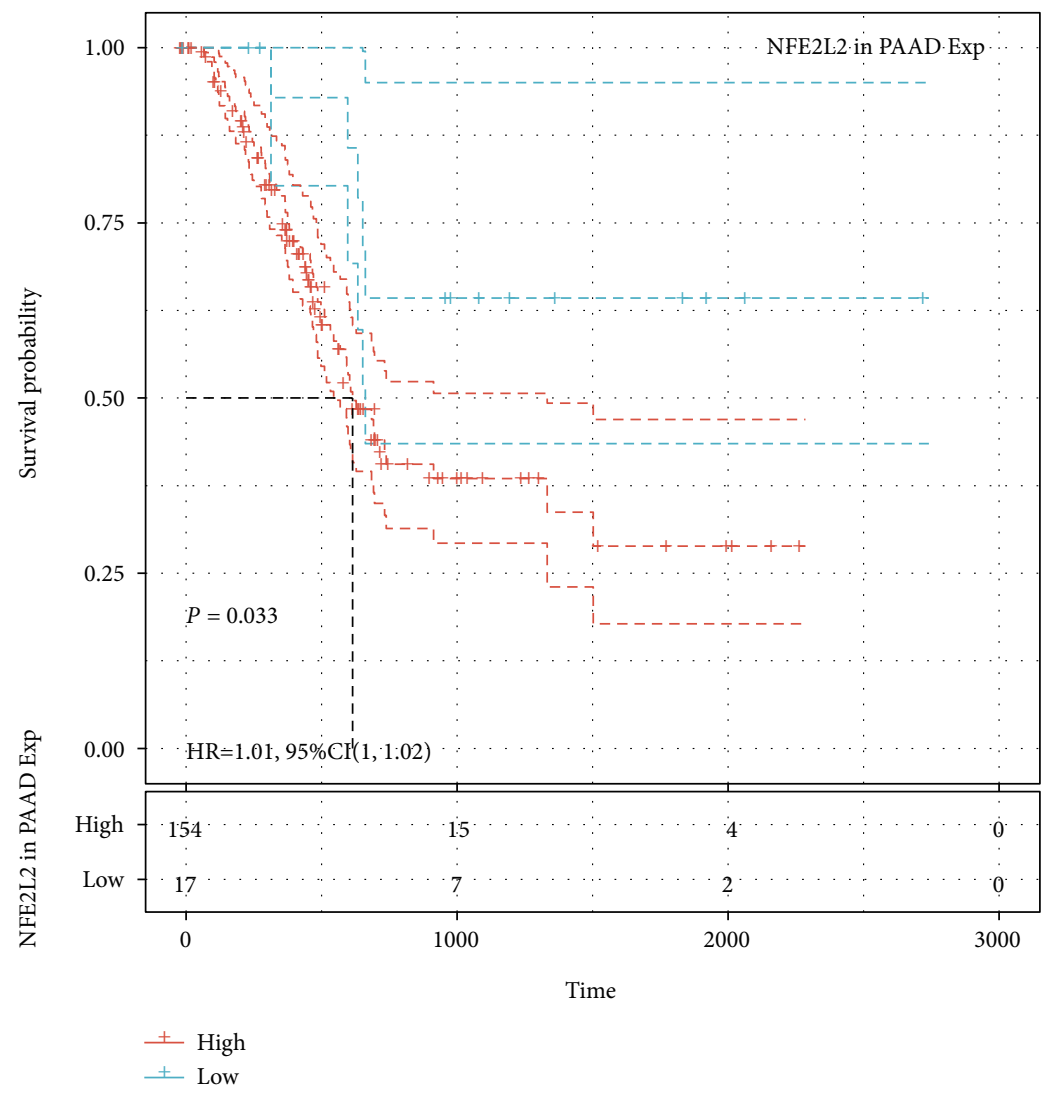

(e)

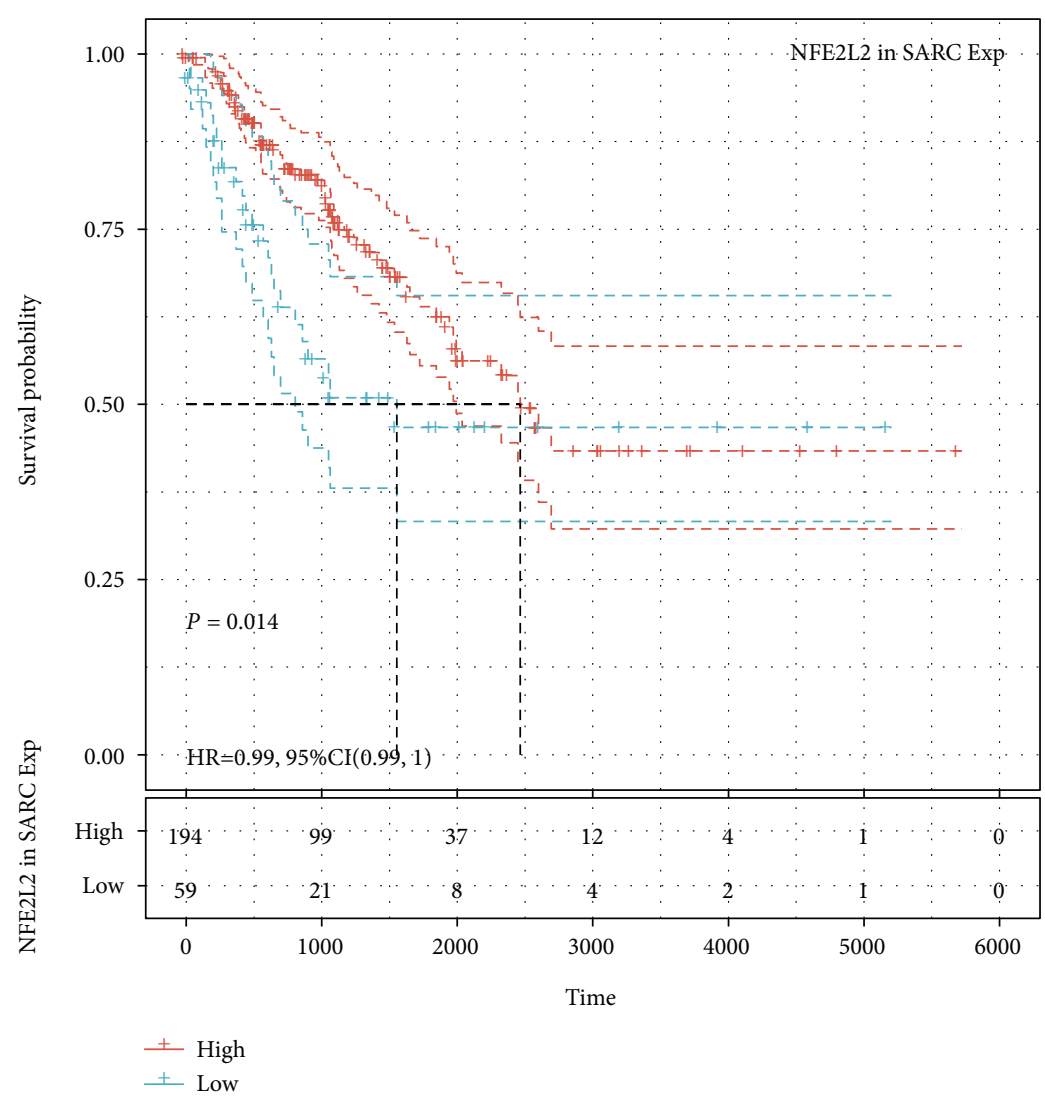

(f)

Figure 4: Continued. 


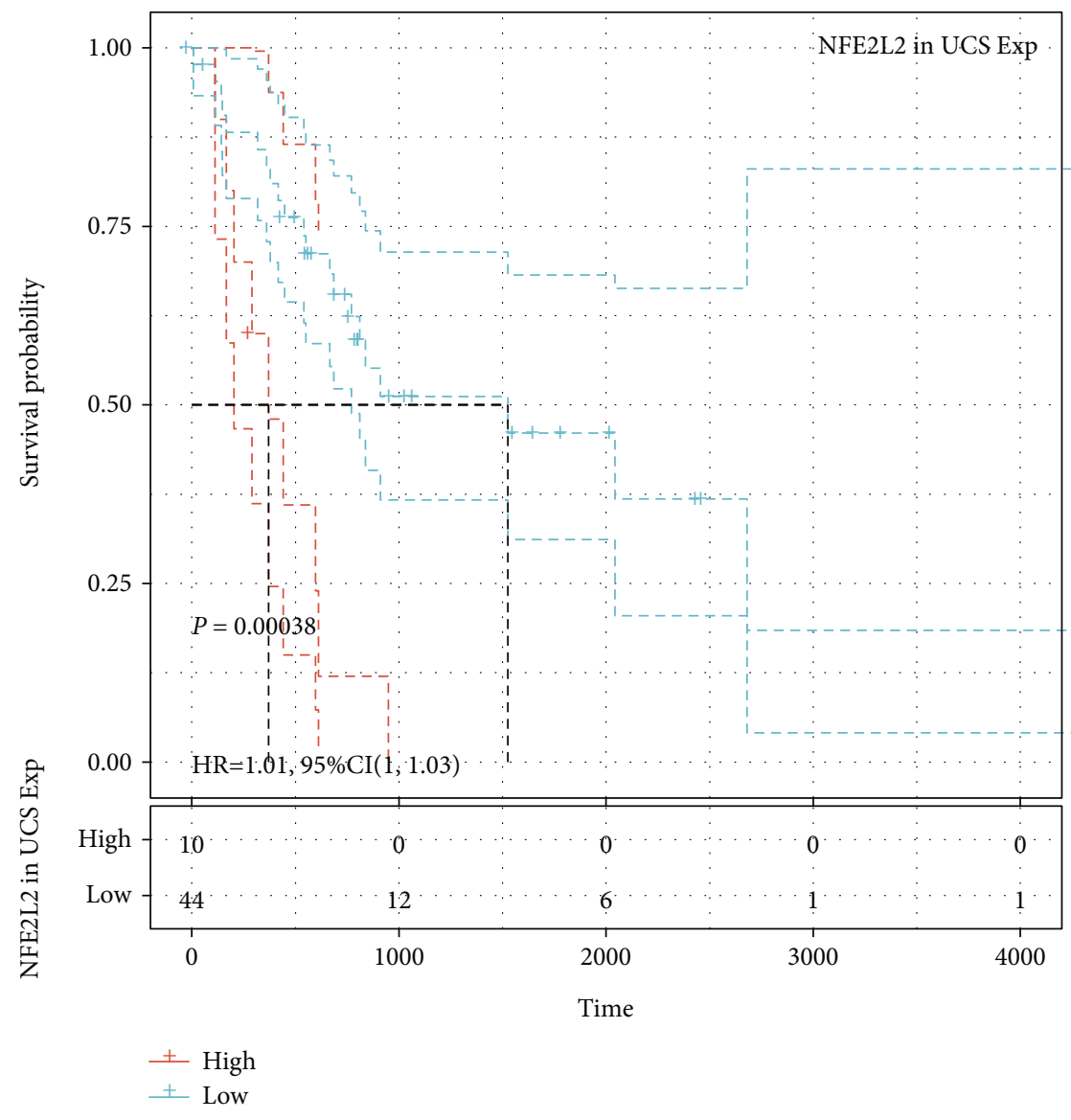

(g)

FIGURE 4: Relationship of NFE2L2 expression with patients' DSS. (a) Forest plots showing the HRs related to NFE2L2 expression in 33 cancer types. (b-g) Kaplan-Meier DSS curves for patients stratified by different expression levels of NFE2L2 in 6 cancer types.

3.2. NFE2L2 Is Correlated with MMR Gene Mutation Levels and DNA Methyltransferase Gene Expression in Human Pan-Cancer. MMRs is a DNA damage repair mechanism. Functional loss of key genes in this mechanism leads to DNA replication errors [24], higher somatic mutations, and tumorigenesis $[22,25]$. To evaluate the role of NFE2L2 in tumorigenesis, we analyzed the correlation between NFE2L2 expression and MMR gene mutation levels. Results showed that NFE2L2 expression was positively related to the mutation levels of 5 MMR genes (MLH1, MSH2, MSH6, PMS2, and EPCAM) in human cancers (Figure 2(a)).

DNA methylation is an epigenetic modification that can alter gene expression [26]. Alteration of the DNA methylation status is an important factor in tumorigenesis [27]. Next, we further evaluated the correlation between NFE2L2 expression and that of 4 DNA methyltransferases. Evidently, NFE2L2 expression is closely related to the expression of DNMT1, DNMT2, DNMT3A, and DNMT3B across human cancers, especially in COAD, KIRP, LGG, and UVM (Figure 2(b)). In summary, these results indicate that NFE2L2 may mediate tumorigenesis by regulating DNA damage or methylation.

3.3. Prognostic Value of NFE2L2 in Human Pan-Cancer. Next, we investigated the relationship between NFE2L2 expression and the prognosis of patients in pan-cancer. Notably, NFE2L2 expression was significantly correlated with patients' OS in 7 types of cancer (ACC, KIRC, LGG, MESO, PAAD, SARC, and UCS) (Figure 3(a)). Specifically, NFE2L2 appeared to be a risk factor in 4 cancer types: $\operatorname{ACC}(P=0.0016, \mathrm{HR}=1.03)$, LGG $(P<0.0001, \mathrm{HR}=1.03)$, PAAD $(P=0.0076, \mathrm{HR}=$ $1.01)$, and UCS $(P=0.00019, \mathrm{HR}=1.02)$. In addition, NFE2L2 was a protective factor in 3 other types of cancer: KIRC $(P<0.0001, \mathrm{HR}=0.99)$, MESO $(P=0.0022, \mathrm{HR}=$ $0.99)$, and SARC $(P=0.0033, \mathrm{HR}=0.99)$ (Figures $3(\mathrm{~b})-$ $3(\mathrm{~h})$ ). Since non-tumor-related factors may cause death during follow-up, we then analyzed the relationship between NFE2L2 expression and DSS in 33 cancers. Results showed NFE2L2 expression impacted patients' DSS in 6 cancer types (ACC, KIRC, LGG, PAAD, SARC, and UCS) (Figure 4(a)). Specifically, Kaplan-Meier curves showed that high expression of NFE2L2 was significantly correlated with poor prognosis of patients in ACC $(P=0.015, \mathrm{HR}=1.02)$, LGG $(P<0.0001$, $\mathrm{HR}=1.03), \quad \mathrm{PAAD} \quad(P=0.033, \mathrm{HR}=1.01)$, and UCS $(P=0.00038, \mathrm{HR}=1.01)$ and reversely in KIRC $(P<0.0001$, $\mathrm{HR}=0.99)$ and SARC $(P=0.014, \mathrm{HR}=0.99)$ (Figures $4(\mathrm{~b})-$ $4(\mathrm{~g}))$. Subsequently, we investigated the relationship between NFE2L2 expression and DFI and found that increased NFE2L2 expression was correlated with poor prognosis in $\operatorname{ACC}(P=0.0021, \mathrm{HR}=1.05)$ and $\operatorname{PAAD}(P=0.026, \mathrm{HR}=$ $1.03)$ but with favorable prognosis in $\mathrm{OV}(P=0.0099, \mathrm{HR}=$ 


\begin{tabular}{|c|c|c|}
\hline & HR & $P$ value \\
\hline $\mathrm{ACC}$ & $1.05(1.02 \sim 1.09)$ & 0.0027 \\
\hline BLCA & $1(0.99 \sim 1.01)$ & 0.6300 \\
\hline BRCA & $1(0.99 \sim 1.01)$ & 0.5000 \\
\hline CESC & $1(1 \sim 1)$ & 0.8500 \\
\hline $\mathrm{CHOL}$ & $0.99(0.97 \sim 1.02)$ & 0.6000 \\
\hline COAD & $1.01(0.99 \sim 1.03)$ & 0.4200 \\
\hline DLBC & $0.99(0.93 \sim 1.05)$ & 0.7100 \\
\hline ESCA & $1(1 \sim 1)$ & 0.6700 \\
\hline GBM & $\mathrm{NA}(\mathrm{NA} \sim \mathrm{NA})$ & \\
\hline HNSC & $1(1 \sim 1.01)$ & 0.9300 \\
\hline $\mathrm{KICH}$ & $1.02(0.96 \sim 1.08)$ & 0.5600 \\
\hline KIRC & $1(0.98 \sim 1.01)$ & 0.8000 \\
\hline KIRP & $1.01(1 \sim 1.02)$ & 0.1100 \\
\hline LAML & $\mathrm{NA}(\mathrm{NA} \sim \mathrm{NA})$ & \\
\hline LGG & $1(0.98 \sim 1.03)$ & 0.7200 \\
\hline LIHC & $1(0.99 \sim 1.01)$ & 0.5500 \\
\hline LUAD & $1(1 \sim 1.01)$ & 0.4700 \\
\hline LUSC & $1(1 \sim 1)$ & 0.5700 \\
\hline MESO & $0.99(0.97 \sim 1.01)$ & 0.3100 \\
\hline OV & $0.99(0.98 \sim 1)$ & 0.0100 \\
\hline PAAD & $1.03(1.01 \sim 1.05)$ & 0.0099 \\
\hline PCPG & $0.99(0.93 \sim 1.06)$ & 0.8500 \\
\hline PRAD & $0.98(0.96 \sim 1)$ & 0.0320 \\
\hline READ & $0.98(0.93 \sim 1.03)$ & 0.4800 \\
\hline SARC & $1(0.99 \sim 1.01)$ & 0.5900 \\
\hline SKCM & NA(NA NA) & \\
\hline STAD & $1(0.99 \sim 1.01)$ & 0.7200 \\
\hline TGCT & $0.99(0.97 \sim 1.02)$ & 0.6500 \\
\hline THCA & $0.99(0.98 \sim 1.01)$ & 0.3400 \\
\hline THYM & $\mathrm{NA}(\mathrm{NA} \sim \mathrm{NA})$ & \\
\hline UCEC & $1.01(1 \sim 1.02)$ & 0.2100 \\
\hline UCS & $1.01(0.98 \sim 1.04)$ & 0.6600 \\
\hline UVM & $\mathrm{NA}(\mathrm{NA} \sim \mathrm{NA})$ & \\
\hline
\end{tabular}

0
0
0
0

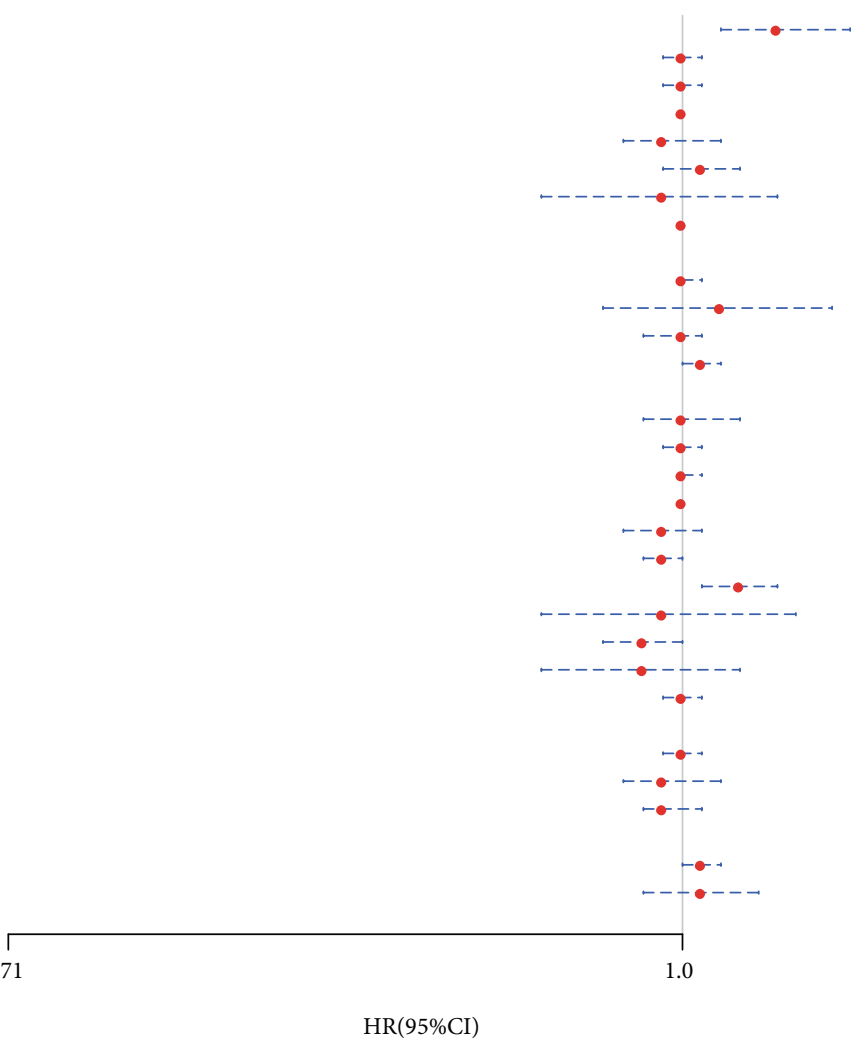

(a)

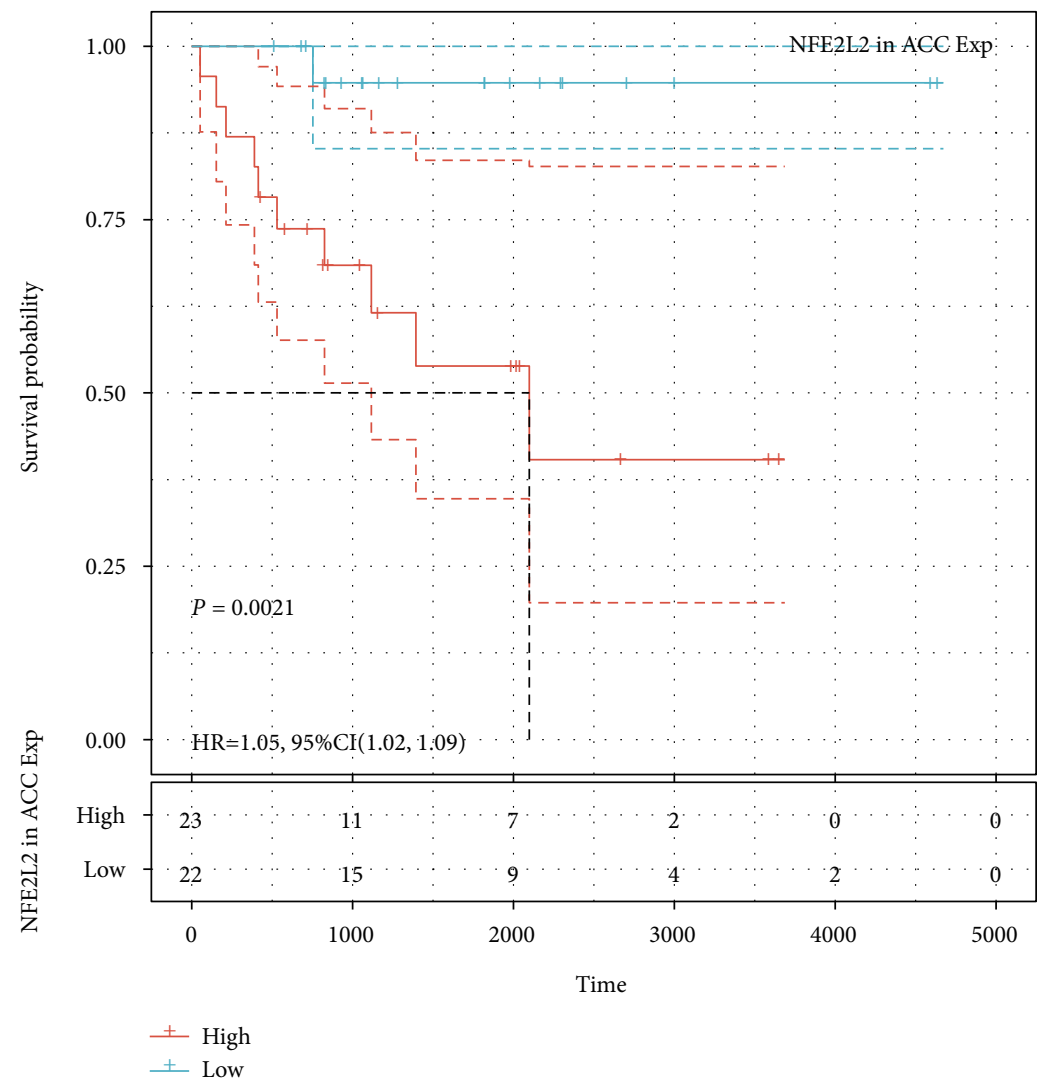

(b)

FIgure 5: Continued. 


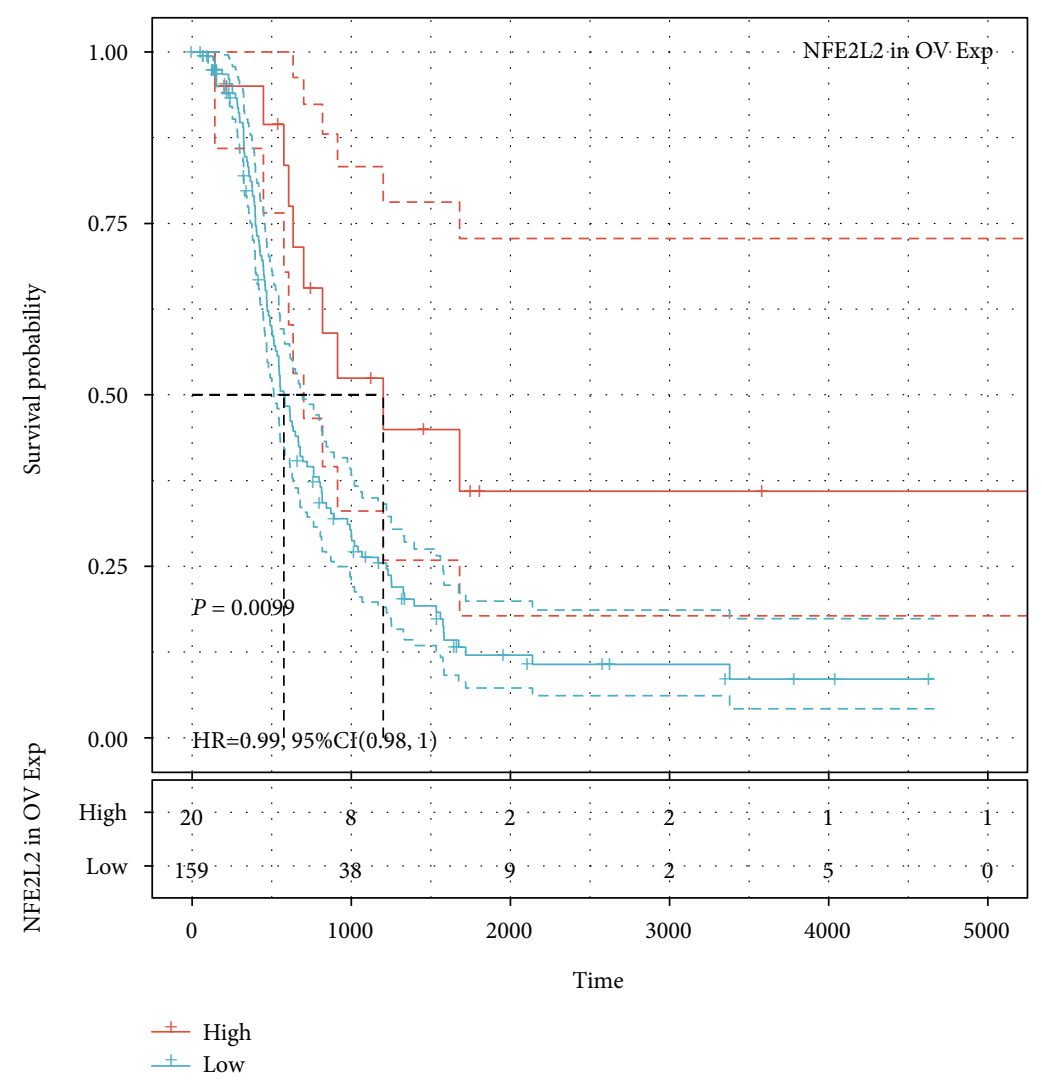

(c)

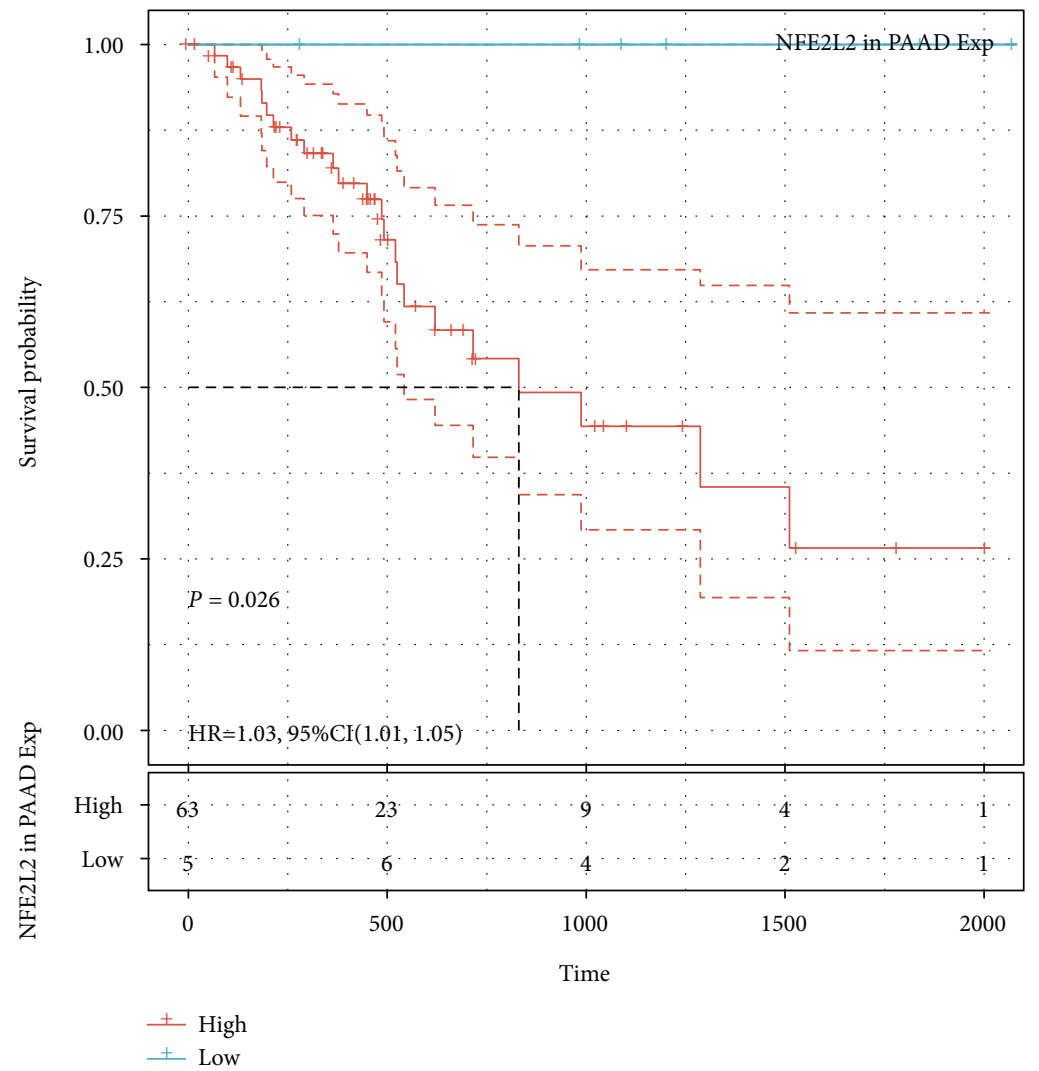

(d)

Figure 5: Continued. 


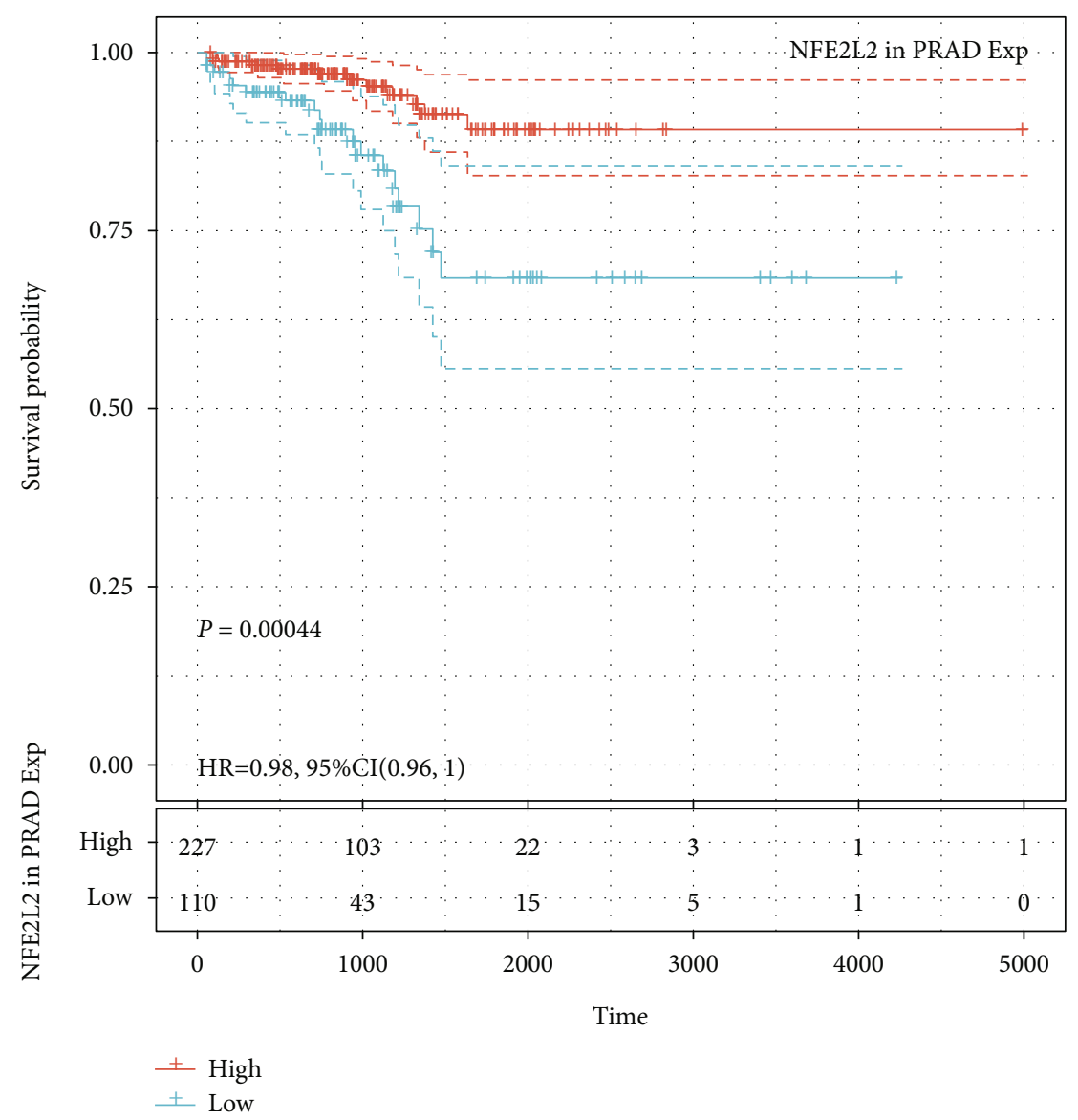

(e)

FIgURE 5: Relationship of NFE2L2 expression with patients' DFI. (a) Forest plots showing the HRs related to NFE2L2 expression in 33 cancer types. (b-e) Kaplan-Meier DFI curves for patients stratified by different expression levels of NFE2L2 in ACC, OV, PAAD, and PRAD.

0.99) and PRAD $(P=0.00044, \mathrm{HR}=0.98)$ (Figure 5). Moreover, we assessed the relationship between NFE2L2 expression and PFI. The results showed that high expression of NFE2L2 affected PFI unfavorably in ACC $(P<0.0001, \mathrm{HR}=1.03)$, LGG $(P<0.0001, \mathrm{HR}=1.02)$, PAAD $(P=0.013$, HR $=1.01)$, and UVM $(P=0.0062, \mathrm{HR}=1.03)$ but favorably in KIRC $(P<0.0001, \mathrm{HR}=0.99)$ and $\operatorname{MESO}(P=8 e-04, \mathrm{HR}=0.99)$ (Figure 6). In conclusion, these results suggest that NFE2L2 expression is significantly correlated with the prognosis of patients, especially those with ACC, LGG, and PAAD.

\subsection{NFE2L2 Expression Is Correlated with Immune Infiltration} Levels and Immune Checkpoint Marker Expression across Cancers. Immune cells in the TME affect patients' survival [28]. Therefore, the correlation between NFE2L2 expression and immune infiltration in human pan-cancer was further studied. First, we downloaded the scores of 6 types of infiltrating immune cells in 33 types of cancer from the TIMER database and then analyzed the correlation between the NFE2L2 expression level and immune infiltration levels. Results showed that NFE2L2 expression was appreciably positively correlated with the infiltration levels of 6 immune cells, including B cells, CD4+ T cells, CD8+ T cells, neutrophils, macrophages, and dendritic cells in LGG, PRAD, KIRC, COAD, and BRCA (Figure 7).
The immune score (i.e., ImmuneScore) and matrix score (i.e., StromalScore) were used to quantify the immune and matrix components in pan-cancer. NFE2L2 expression was positively correlated with the ImmuneScore in DLBC, LGG, PAAD, PRAD, LAML, and negatively correlated with the ImmuneScore in ESCA, LUSC, THYM, THCA, and MESO (Figure 8(a)). In addition, NFE2L2 expression was positively correlated with the StromalScore in LAML, LGG, BRCA, TGCT, DLBC, PAAD, PCPG, PRAD, and THYM and negatively correlated with the StromalScore in LUSC (Figure 8(b)).

Immune checkpoint inhibitors (ICIs), as novel tumor immunotherapy agents, play an important role in tumor immunotherapy [29]. Subsequently, we analyzed the correlation between NFE2L2 expression and that of 40 common immune checkpoint genes. Interestingly, in LGG and PRAD, NFE2L2 expression was correlated with more than 30 immune checkpoint markers, such as TNFSF4, CD48, and CD28 (Figure 9). Collectively, these results strongly suggest that NFE2L2 plays a vital role in tumor immunity.

\section{Discussion}

Pan-cancer analysis can reveal similarities and differences in tumors, providing insights into cancer prevention and the design of therapeutic targets [30]. Recently, many studies 


$\begin{array}{lrl} & \text { HR } & P \text { value } \\ \text { ACC } & 1.03(1.02 \sim 1.05) & 5.2 \mathrm{e}-05 \\ \text { BLCA } & 1(1 \sim 1) & 9.1 \mathrm{e}-01 \\ \text { BRCA } & 1(0.99 \sim 1) & 4.1 \mathrm{e}-01 \\ \text { CESC } & 1(0.99 \sim 1) & 2.1 \mathrm{e}-01 \\ \text { CHOL } & 0.99(0.97 \sim 1.01) & 3.4 \mathrm{e}-01 \\ \text { COAD } & 1(0.99 \sim 1.01) & 8.3 \mathrm{e}-01 \\ \text { DLBC } & 1(0.97 \sim 1.03) & 9.7 \mathrm{e}-01 \\ \text { ESCA } & 1(1 \sim 1) & 7.2 \mathrm{e}-01 \\ \text { GBM } & 1(1 \sim 1.01) & 3.9 \mathrm{e}-01 \\ \text { HNSC } & 1(1 \sim 1) & 8.2 \mathrm{e}-01 \\ \text { KICH } & 1(0.98 \sim 1.02) & 6.9 \mathrm{e}-01 \\ \text { KIRC } & 0.99(0.99 \sim 1) & 1.9 \mathrm{e}-03 \\ \text { KIRP } & 1.01(1 \sim 1.01) & 6.1 \mathrm{e}-02 \\ \text { LAML } & \mathrm{NA}(\mathrm{NA} \sim \mathrm{NA}) & \\ \text { LGG } & 1.02(1.01 \sim 1.02) & 3.1 \mathrm{e}-08 \\ \text { LIHC } & 1.01(1 \sim 1.01) & 1.9 \mathrm{e}-01 \\ \text { LUAD } & 1(1 \sim 1) & 5.9 \mathrm{e}-01 \\ \text { LUSC } & 1(1 \sim 1) & 6.1 \mathrm{e}-01 \\ \text { MESO } & 0.99(0.98 \sim 1) & 1.5 \mathrm{e}-02 \\ \text { OV } & 1(0.99 \sim 1) & 5.4 \mathrm{e}-02 \\ \text { PAAD } & 1.01(1 \sim 1.02) & 1.9 \mathrm{e}-02 \\ \text { PCPG } & 1(0.98 \sim 1.03) & 8.1 \mathrm{e}-01 \\ \text { PRAD } & 0.99(0.98 \sim 1) & 1.8 \mathrm{e}-01 \\ \text { READ } & 0.99(0.98 \sim 1.01) & 4.1 \mathrm{e}-01 \\ \text { SARC } & 1(0.99 \sim 1) & 7.1 \mathrm{e}-02 \\ \text { SKCM } & 1(0.99 \sim 1) & 2.2 \mathrm{e}-01 \\ \text { STAD } & 1(0.99 \sim 1.01) & 6.2 \mathrm{e}-01 \\ \text { TGCT } & 1(0.98 \sim 1.02) & 7.1 \mathrm{e}-01 \\ \text { THCA } & 1(0.99 \sim 1) & 2.9 \mathrm{e}-01 \\ \text { THYM } & 1(0.98 \sim 1.01) & 4.0 \mathrm{e}-01 \\ \text { UCEC } & 1(1 \sim 1.01) & 2.5 \mathrm{e}-01 \\ \text { UCS } & 1.01(1 \sim 1.02) & 1.2 \mathrm{e}-01 \\ \text { UVM } & 1.03(1.01 \sim 1.05) & 6.3 \mathrm{e}-03 \\ & & \end{array}$

\section{$\operatorname{HR}(95 \% \mathrm{CI})$}

(a)

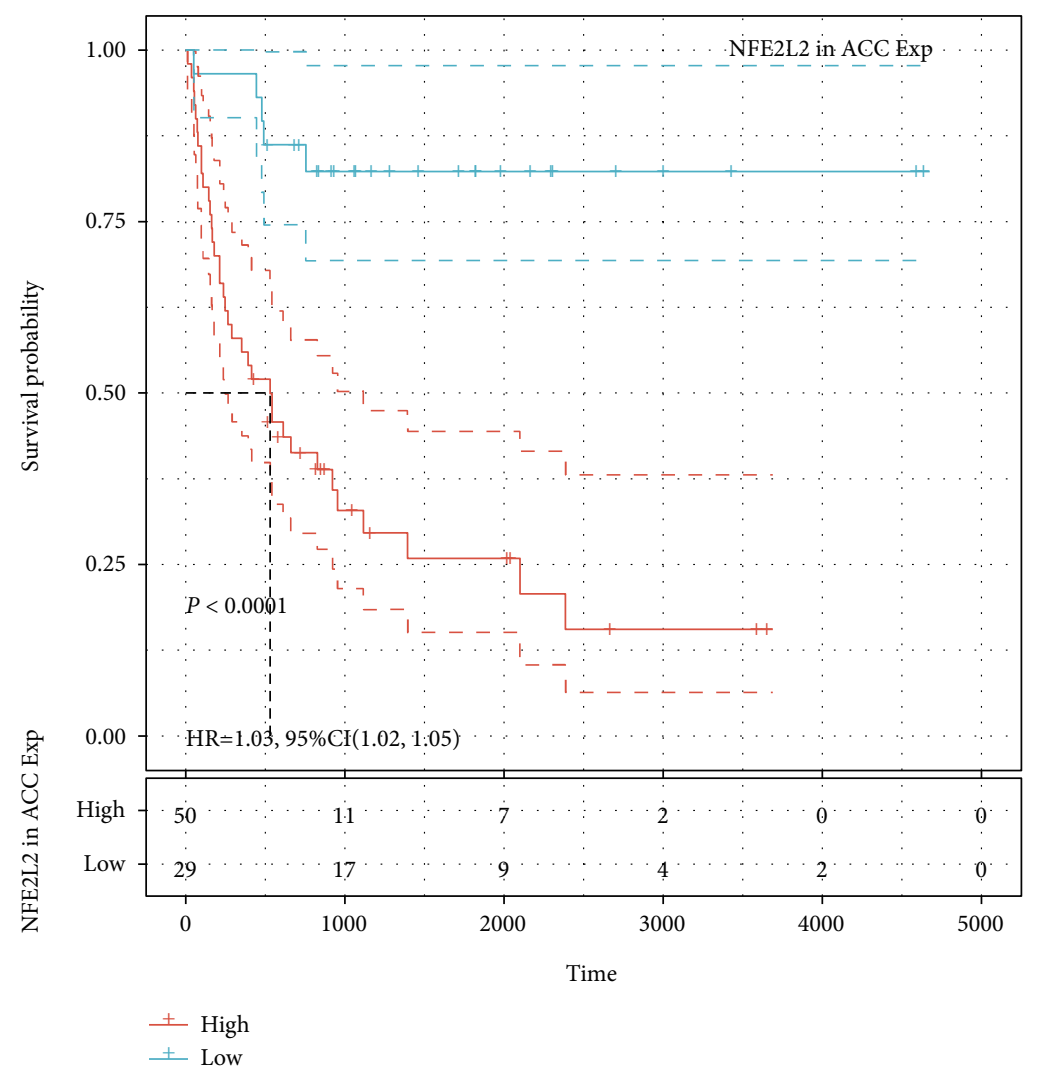

(b)

Figure 6: Continued. 


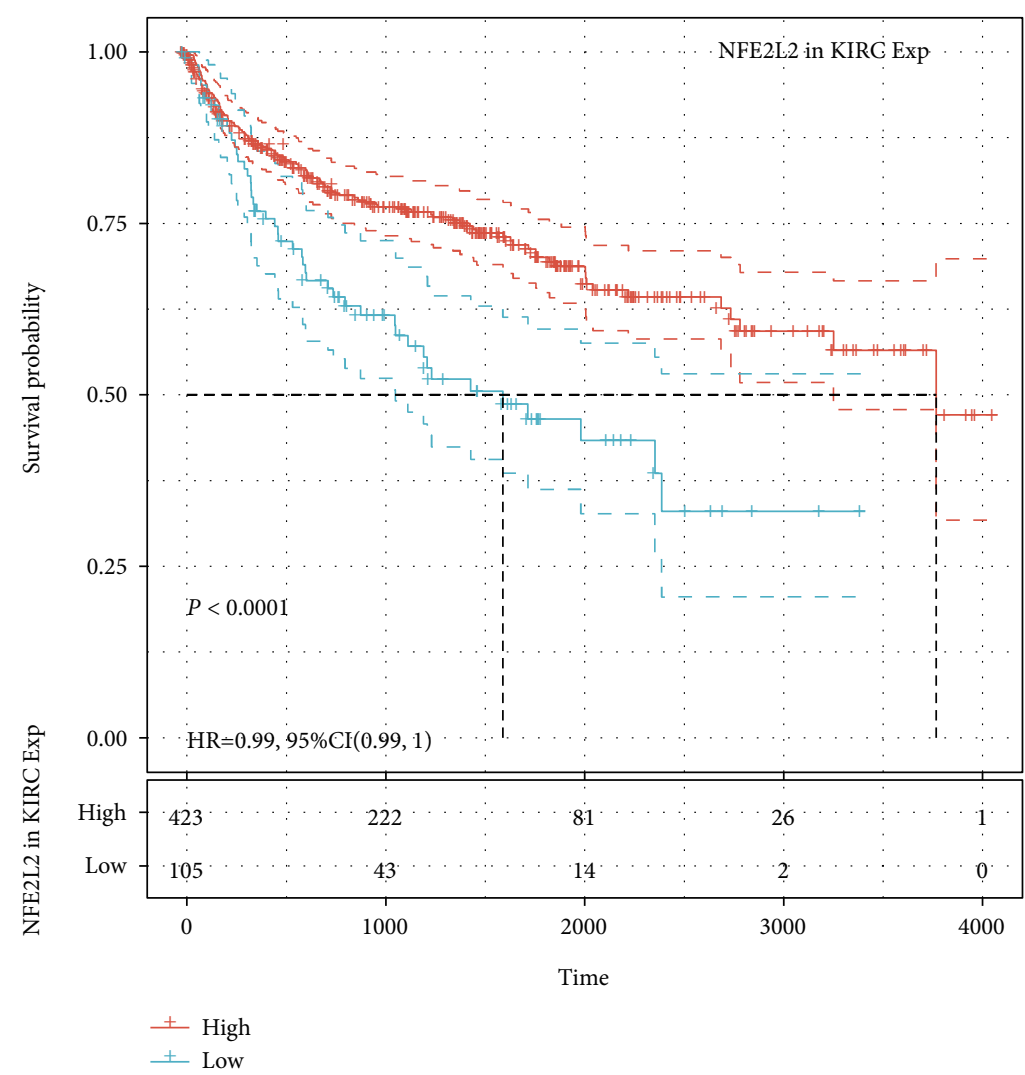

(c)

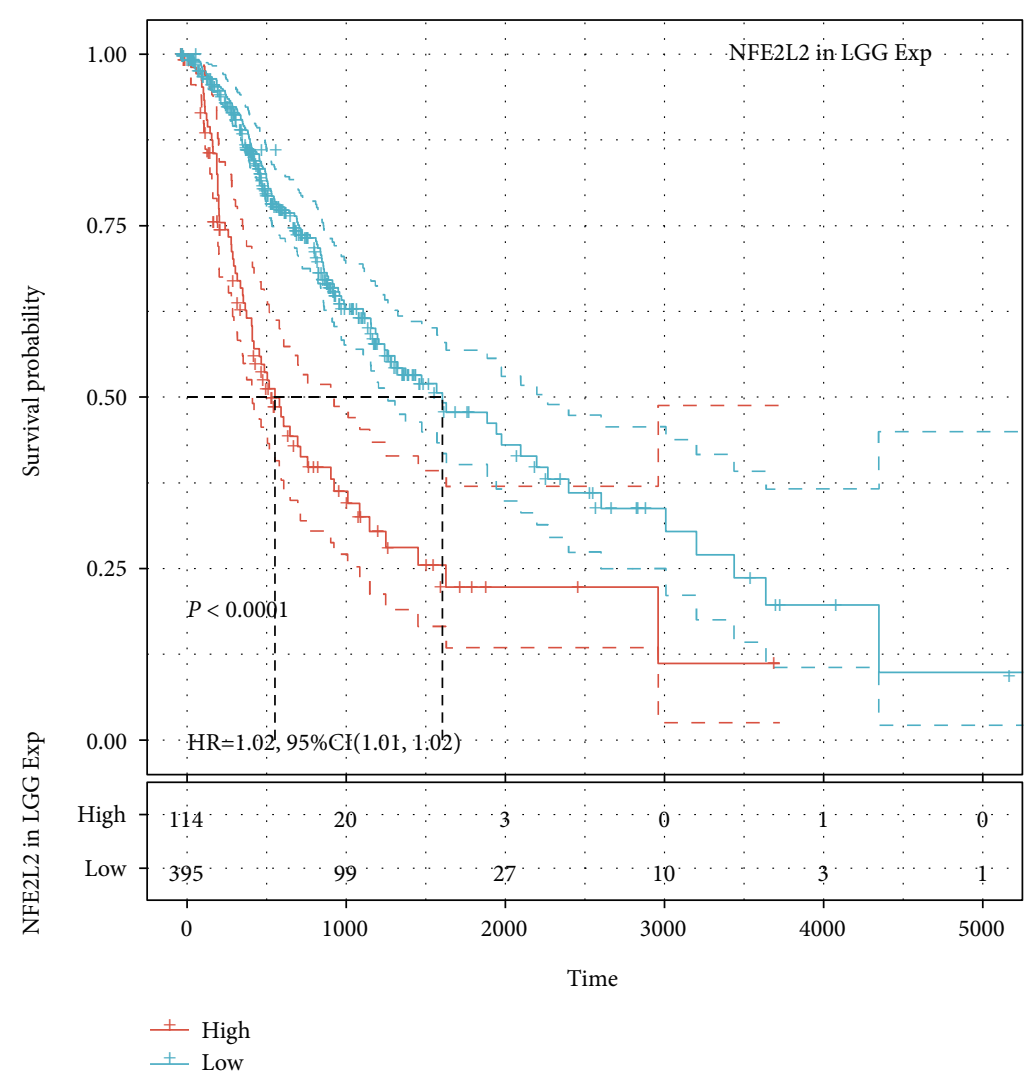

(d)

Figure 6: Continued. 


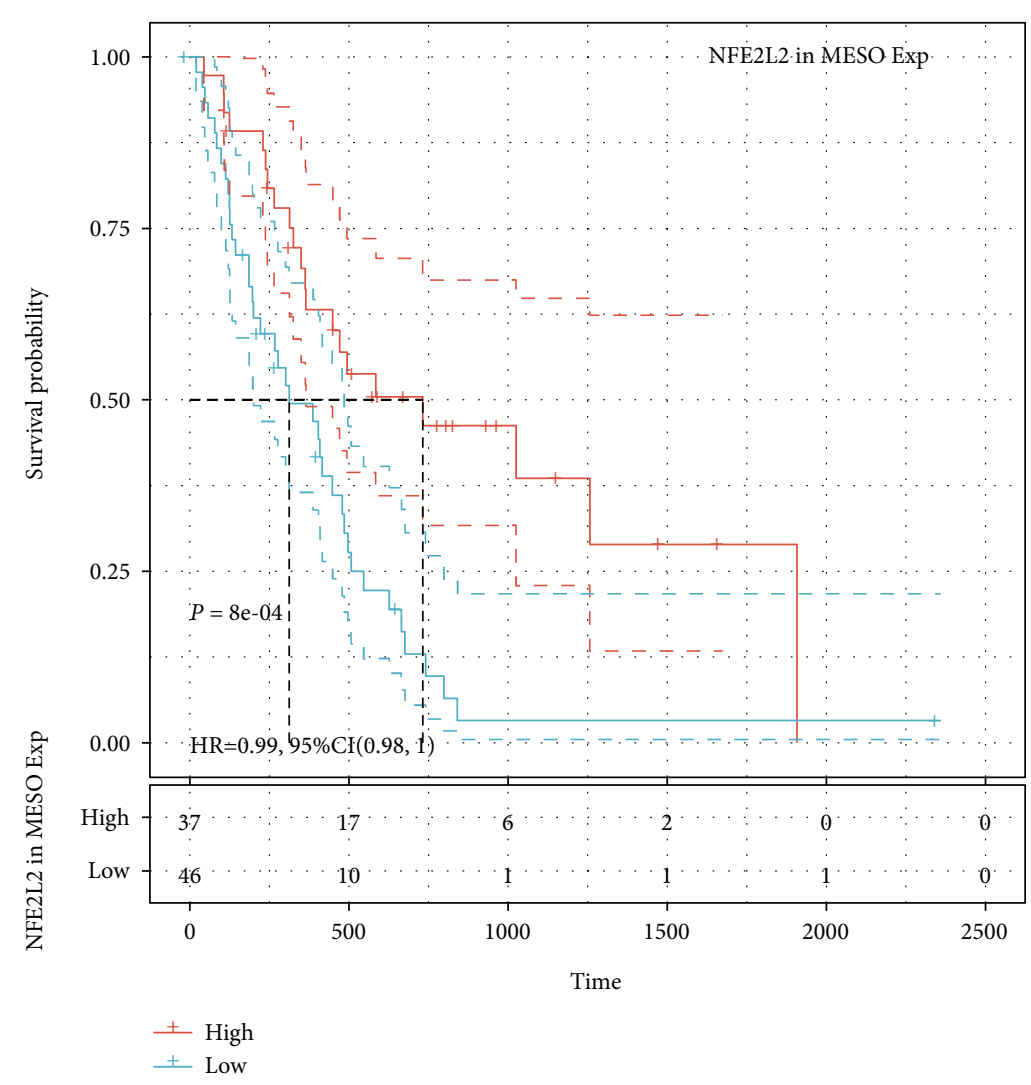

(e)

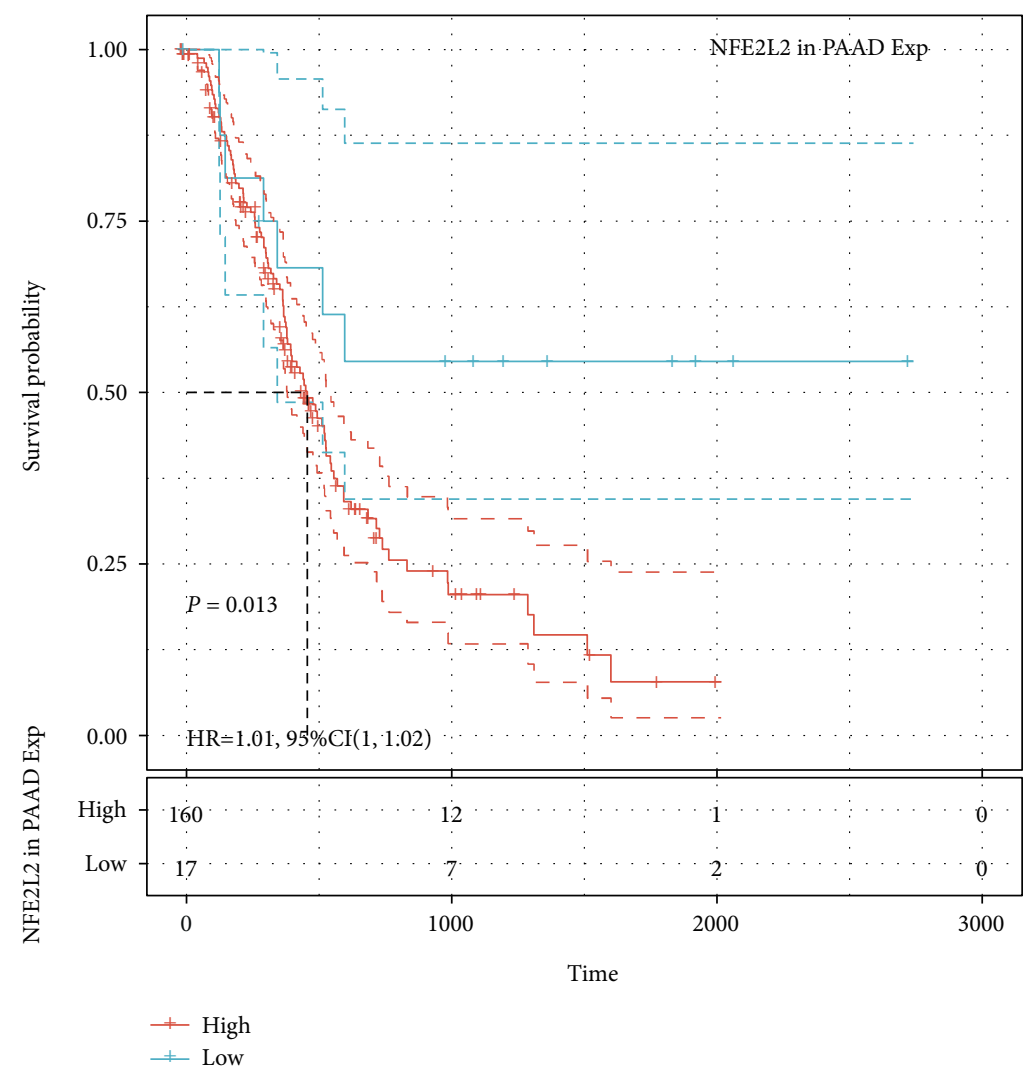

(f)

FIgure 6: Continued. 


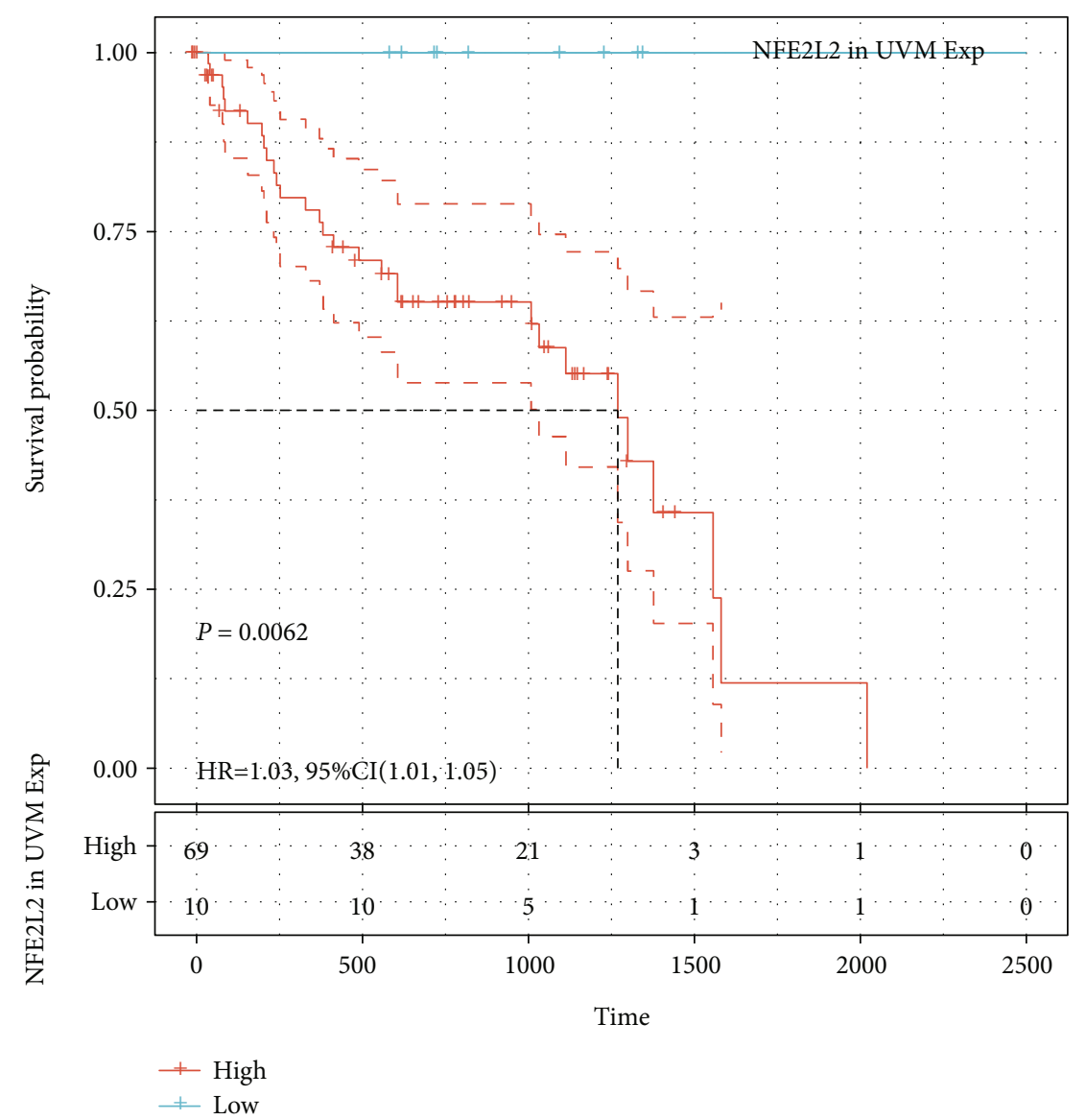

(g)

FIgURE 6: Relationship of NFE2L2 expression with patients' PFI. (a) Forest plots showing the HRs related to NFE2L2 expression in 33 cancer types. (b-g) Kaplan-Meier PFI curves for patients stratified by different expression levels of NFE2L2 in 6 cancer types.

have focused on pan-cancer analysis of the whole genome, revealing mutations, RNA alterations, and driver genes that are related to the occurrence and development of cancer, which is of importance for early diagnosis of cancer and development of biomarkers [31-35]. NFE2L2 is a transcription factor with alkaline lysine zipper structure, which plays a role in resisting oxidative stress and maintaining the body's redox homeostasis [36]. However, the roles of NFE2L2 in human pan-cancer have not been identified, and whether it can be used as a biomarker is still unknown. In the current study, we found that NFE2L2 is abnormally expressed in 22 cancer types and is significantly correlated with MMR gene mutation levels and DNA methylation. In addition, NFE2L2 expression was associated with poor prognosis (OS, DSS, and PFI) of patients, especially those with ACC, LGG, and PAAD. Furthermore, we observed that NFE2L2 expression was positively correlated with immune infiltration levels and the expression of immune checkpoint markers, especially in LGG. The above results strongly suggested that NFE2L2 may be used as a potential biomarker of LGG and play an indispensable role in tumor immunity.

Studies have shown that NFE2L2 could bind to KEAP1, which acts as a redox sensor to dissociate NFE2L2 from its cytoplasmic complex for translocation into the nucleus [37, 38]. In the nucleus, NFE2L2 binds to the antioxidant response element (ARE) to activate the expression of detoxification, antioxidant, and anti-inflammatory genes, establishing the NFE2L2/KEAP1/ARE signaling pathway [37]. Disrupting the balance of this pathway can lead to aging, inflammation, and tumor chemoresistance $[39,40]$. In addition, several studies have indicated that NFE2L2 is upregulated in different types of cancers and correlates with tumor progression, aggressiveness, and poor prognosis [41]. Another study showed that cytoplasmic NFE2L2 expression was associated with patients poor prognosis, while the nuclear NFE2L2 expression was associated with a more favorable prognosis [42]. Moreover, NFE2L2 is abnormally overexpressed in lung cancer cell line A549 [43]. These previous findings indicate NFE2L2 may be abnormally expressed in various cancers and play important roles in cancer progression and patients' prognosis. In this study, we found for the first time that abnormal expression of NFE2L2 exists in human pan-cancer including ACC, LGG, and PAAD. Survival analysis showed NFE2L2 expression was associated with poor prognosis in multiple cancers, especially in ACC, LGG, and PAAD. These results strongly indicate NFE2L2 is a potential prognostic biomarker in ACC, LGG, and PAAD.

Under normal conditions, MMRs ensures the stability of DNA replication. MMRs consists of multiple heterodimers, including MLH1/PMS2, MSH2/MSH6, and EPCAM, 

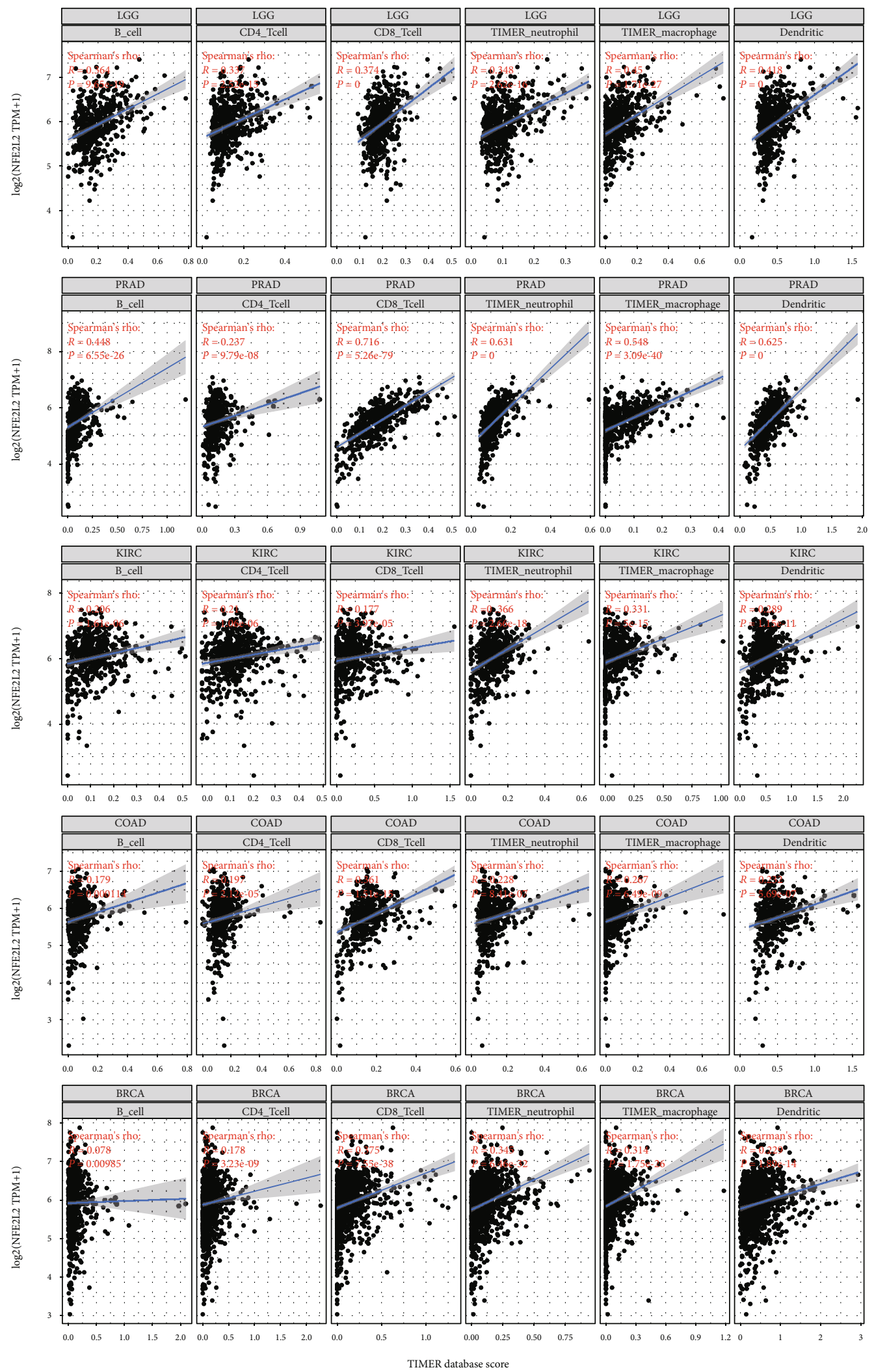

FIGURE 7: Correlation of NFE2L2 expression with immune infiltration levels of B cells, CD4+ T cells, CD8+ T cells, neutrophil cells, macrophage cells, and dendritic cells. 

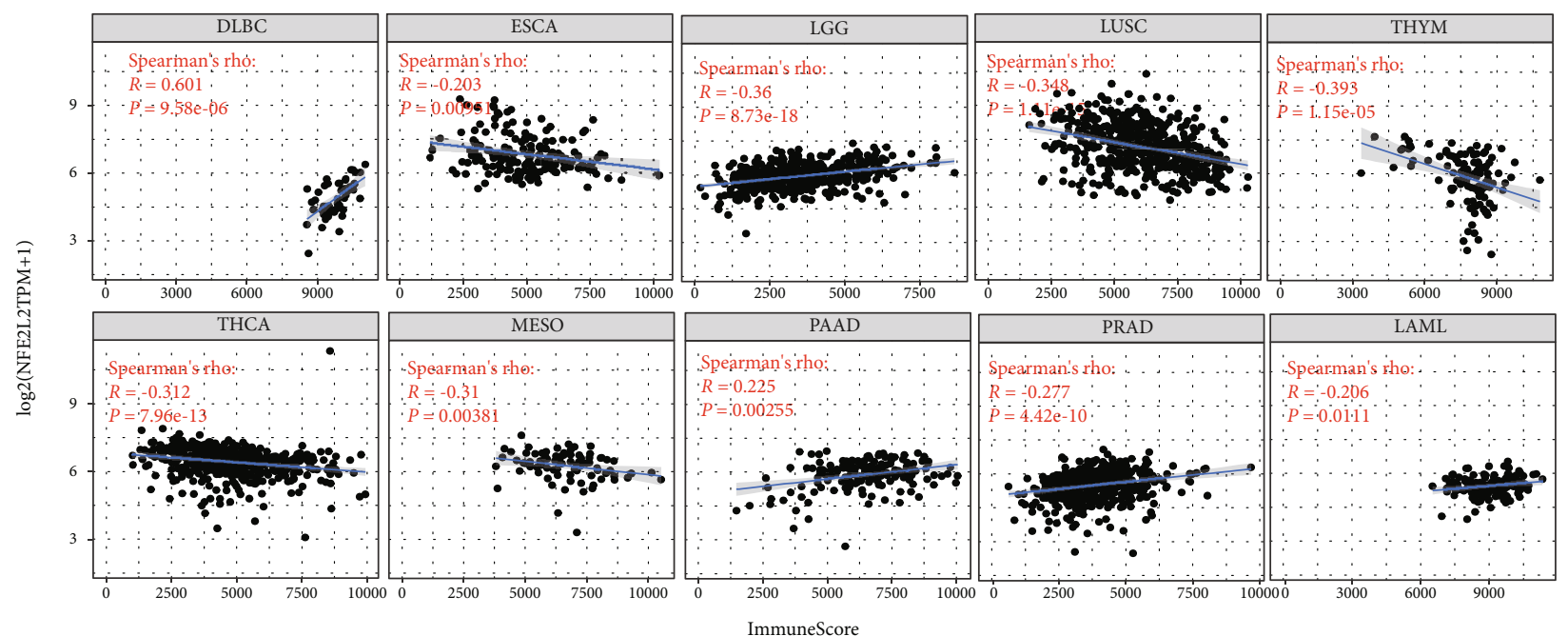

(a)
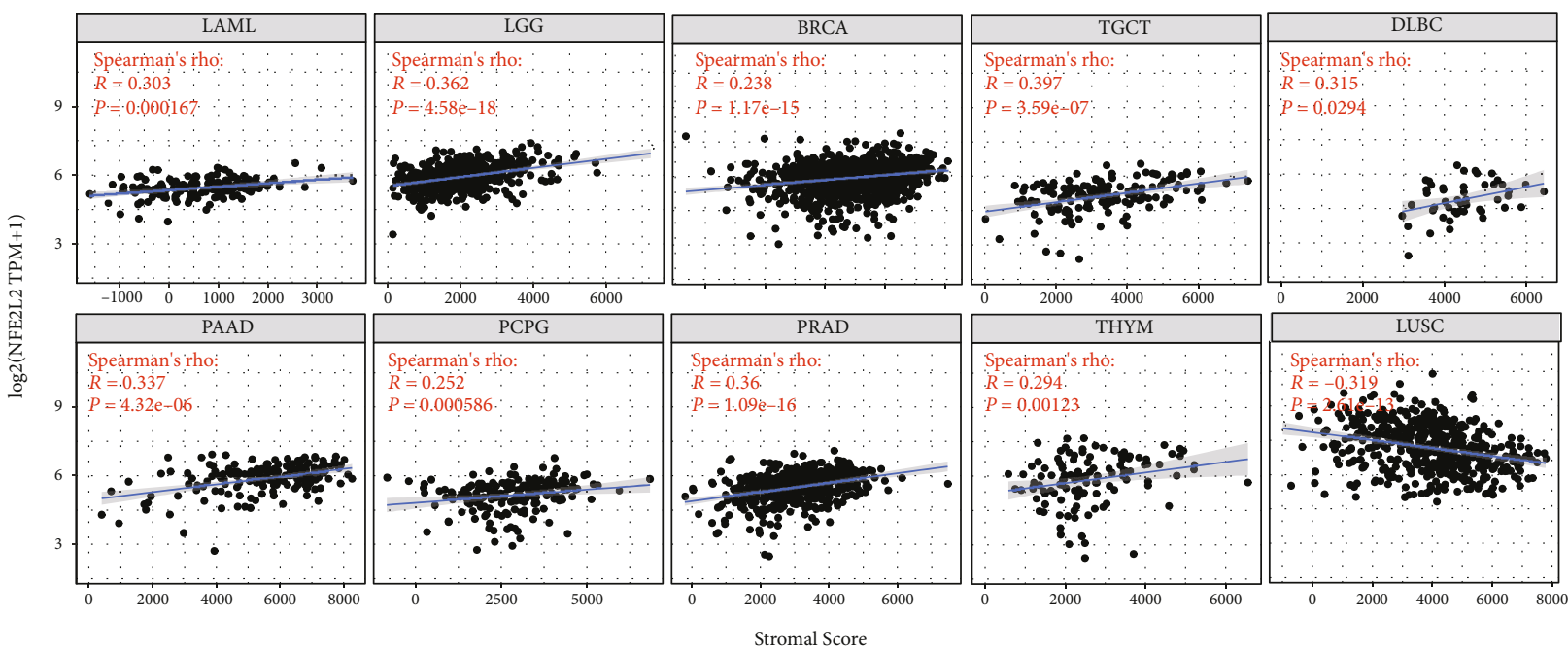

(b)

FIGURE 8: Correlation analysis between NFE2L2 expression and ImmuneScore/Stromal Score in cancers. (a) Correlation between NFE2L2 expression and ImmuneScore in DLBC, ESCA, LGG, LUSC, THYM, THCA, MESO, PAAD, PRAD, and LAML. (b) Correlation between NFE2L2 expression and StromalScore in LAML, LGG, BRCA, TGCT, DLBC, PAAD, PCPG, PRAD, THYM, and LUSC.

which can identify and correct gene mutations including base substitutions, insertions, deletions, or mismatches during DNA replication [44]. Mutations or defects in the MMR gene can lead to the accumulation of genetic errors, resulting in genomic or microsatellite instability, which contribute to the occurrence of tumors [45]. These indicate MMR gene mutation is a predictor of tumorigenesis. In this study, through correlation analysis, we found NFE2L2 expression was closely associated with the mutation levels of 5 MMR genes (MLH1, MSH2, MSH6, PMS2, and EPCAM) in human pan-cancer. In addition, alterations in DNA methylation status contribute to the development of cancer [46]. Recent research has shown that hypermethylation of the gene promoter is a common epigenetic feature of cancer [47, 48]. In our study, we also found that NFE2L2 expression was closely correlated with that of 4 DNA methyltransferases (DNMT1, DNMT2, DNMT3A, and DNMT3B) in human cancers, especially in
COAD, KIRP, LGG, and UVM. These results strongly support our conclusion that abnormal expression of NFE2L2 may play an important role in tumorigenesis by regulating MMR gene mutation levels and DNA methylation.

The TME has been a recent focus of tumor research. The immune microenvironment composed of tumor-infiltrating lymphocytes (TILs; B cells and T cells) and other immune cells (dendritic cells, neutrophils, and macrophages) is an important part of the TME $[49,50]$. Studies have shown that immune cells play an indispensable role as a double-edged sword in tumors to promote or inhibit tumor progression [51-53]. Under normal conditions, immune cells play an antitumor role by monitoring and destroying cancer cells [54]. On the other hand, studies have shown that cancer cells can evade the surveillance of immune cells through a variety of mechanisms [55-58]. TILs have been shown to be an independent predictor of patients' prognosis in cancers [59]. CD4+ and CD8+ T cells are crucial members of the 


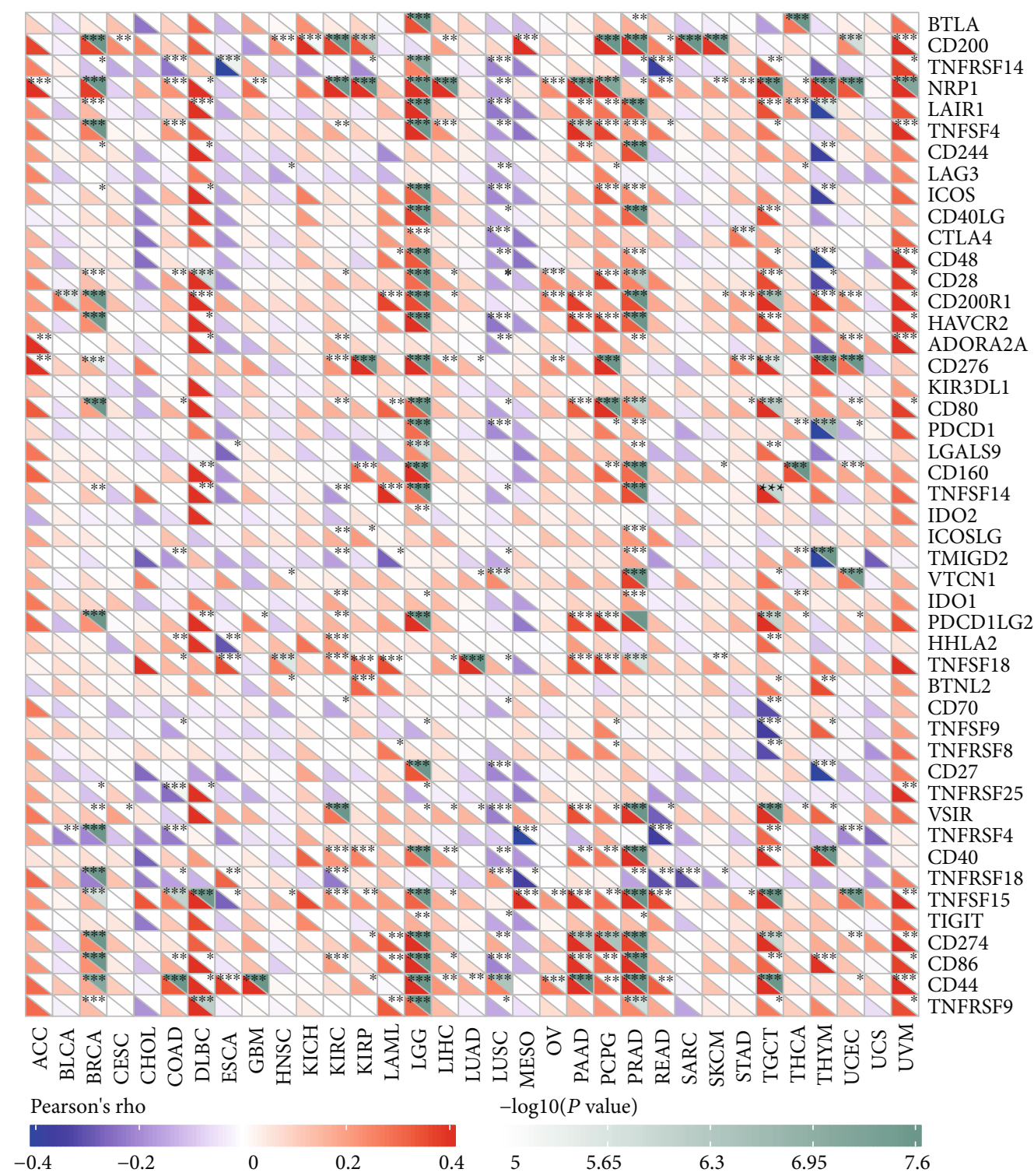

FiguRE 9: Correlation analysis of NFE2L2 expression levels with 40 common immune checkpoint gene levels in pan-cancer.

TME that participate in specific antitumor immune responses [60]. Neutrophils secrete MMP9 into the TME, which contributes to angiogenesis, tumor progression, and metastasis in mouse transplantation models [61]. Macrophages are the first line of defense against tumor immunity. Instead of killing tumor cells, TAMs mediate tumor development [62]. These observations indicate that TILs play a crucial part in tumor progression. However, there are few studies about the roles of NFE2L2 in the immune microenvironment. In this study, we found that NFE2L2 expression was significantly correlated with the levels of 6 types of infiltrating immune cells (B cells, CD4+ T cells, CD8+ T cells, dendritic cells, macrophages, and neutrophils) in BRCA, COAD, KIRC, LGG, and PRAD. These results indicate that NFE2L2 may lead to tumorigenesis or inhibit tumor progression by changing the TIL status. These novel findings constitute substantial progress in identifying the important role of NFE2L2 in immune infiltration.
Immune scoring is an approach to evaluate the infiltrating $\mathrm{CD} 3+/ \mathrm{CD} 45 \mathrm{RO}+, \mathrm{CD} 3+/ \mathrm{CD} 8+$, or $\mathrm{CD} 8+/ \mathrm{CD} 45 \mathrm{RO}+$ lymphocyte population at the center and edges of a tumor [63]. In the TME, a higher ImmuneScore or StromalScore indicates a larger number of immune or matrix components [64]. Our results revealed that NFE2L2 expression was positively correlated with the ImmuneScore in DLBC, LGG, LAML, PAAD, and PRAD and negatively correlated with the ImmuneScore in ESCA, LUSC, THYM, THCA, and MESO. In addition, NFE2L2 expression was positively correlated with the StromalScore in BRCA, DLBC, LAML, LGG, PAAD, PCPG, PRAD, TGCT, and THYM and negatively correlated with the StromalScore in LUSC. Moreover, the correlation between NFE2L2 expression and immune checkpoint markers implies the role of NFE2L2 in regulating tumor immunology in cancers, especially in LGG. These results further strongly indicate NFE2L2's important roles in tumor immunity. 


\section{Conclusions}

In conclusion, the results of the present study indicated that NFE2L2 overexpression correlates with poor prognosis of patients and increases the infiltration levels of $\mathrm{B}$ cells, CD8+ T cells, CD4+ T cells, macrophages, neutrophils, and dendritic cells in many cancers, especially in LGG. In addition, NFE2L2 expression was found to be significantly correlated with the expression of immune checkpoint markers in LGG. Therefore, NFE2L2 may play a vital role in immune infiltration and be a potential prognostic biomarker for LGG.

\section{Data Availability}

The data used to support the findings of this study are available from the corresponding author upon request.

\section{Conflicts of Interest}

The authors declare no conflicts.

\section{Authors' Contributions}

Qiang Ju and Xinmei Li contributed equally to this article.

\section{Acknowledgments}

The authors sincerely thank all participants involved in this study. This study was supported by the National Science Foundation for Young Scientists of China (grant no. 81802415 to YZ), the Shandong Provincial Natural Science Foundation (grant no. ZR2018PH025 to YZ), and the Doctoral Scientific Fund Project of the Affiliated Hospital of Qingdao University (grant no. 2796 to QJ).

\section{References}

[1] M. Hämäläinen, H.-R. Teppo, S. Skarp et al., "NRF1 and NRF2 mRNA and protein expression decrease early during melanoma carcinogenesis: an insight into survival and microRNAs," Oxidative Medicine and Cellular Longevity, vol. 2019, Article ID 2647068, 15 pages, 2019.

[2] S. Ma, C. Paiboonrungruan, T. Yan, K. P. Williams, M. B. Major, and X. L. Chen, "Targeted therapy of esophageal squamous cell carcinoma: the NRF2 signaling pathway as target," Annals of the New York Academy of Sciences, vol. 1434, no. 1, pp. 164-172, 2018.

[3] G. Kontostathi, J. Zoidakis, M. Makridakis et al., "Cervical cancer cell line secretome highlights the roles of transforming growth factor-beta-induced protein ig-h3, peroxiredoxin-2, and NRF2 on cervical carcinogenesis," BioMed Research International, vol. 2017, Article ID 4180703, 15 pages, 2017.

[4] L. Zhang, N. Wang, S. Zhou, W. Ye, G. Jing, and M. Zhang, "Propofol induces proliferation and invasion of gallbladder cancer cells through activation of Nrf2," Journal of Experimental \& Clinical Cancer Research, vol. 31, no. 1, p. 66, 2012.

[5] Y. Mitsuishi, K. Taguchi, Y. Kawatani et al., "Nrf2 redirects glucose and glutamine into anabolic pathways in metabolic reprogramming," Cancer Cell, vol. 22, no. 1, pp. 66-79, 2012.
[6] T. W. Kensler and N. Wakabayashi, "Nrf2: friend or foe for chemoprevention?," Carcinogenesis, vol. 31, no. 1, pp. 90-99, 2010.

[7] A. Singh, S. Boldin-Adamsky, R. K. Thimmulappa et al., "RNAi-mediated silencing of nuclear factor erythroid-2related factor 2 gene expression in non-small cell lung cancer inhibits tumor growth and increases efficacy of chemotherapy," Cancer Research, vol. 68, no. 19, pp. 7975-7984, 2008.

[8] J. Q. Ma, H. Tuersun, S. J. Jiao, J. H. Zheng, J. B. Xiao, and A. Hasim, "Functional role of NRF2 in cervical carcinogenesis," PLoS One, vol. 10, no. 8, article e0133876, 2015.

[9] P. A. Stewart, E. A. Welsh, R. J. C. Slebos et al., "Proteogenomic landscape of squamous cell lung cancer," Nature Communications, vol. 10, no. 1, p. 3578, 2019.

[10] D. R. Powell and A. Huttenlocher, "Neutrophils in the tumor microenvironment," Trends in Immunology, vol. 37, no. 1, pp. 41-52, 2016.

[11] M. Dysthe and R. Parihar, "Myeloid-derived suppressor cells in the tumor microenvironment," Advances in Experimental Medicine and Biology, vol. 1224, pp. 117-140, 2020.

[12] T. F. Gajewski, H. Schreiber, and Y. X. Fu, "Innate and adaptive immune cells in the tumor microenvironment," Nature Immunology, vol. 14, no. 10, pp. 1014-1022, 2013.

[13] S. L. Topalian, C. G. Drake, and D. M. Pardoll, "Immune checkpoint blockade: a common denominator approach to cancer therapy," Cancer Cell, vol. 27, no. 4, pp. 450-461, 2015.

[14] D. F. Quail and J. A. Joyce, "Microenvironmental regulation of tumor progression and metastasis," Nature Medicine, vol. 19, no. 11, pp. 1423-1437, 2013.

[15] Y. Zhu, J. Yang, D. Xu et al., "Disruption of tumour-associated macrophage trafficking by the osteopontin-induced colonystimulating factor-1 signalling sensitises hepatocellular carcinoma to anti-PD-L1 blockade," Gut, vol. 68, no. 9, pp. 16531666, 2019.

[16] M. De Palma, D. Biziato, and T. V. Petrova, "Microenvironmental regulation of tumour angiogenesis," Nature Reviews. Cancer, vol. 17, no. 8, pp. 457-474, 2017.

[17] X. Li, W. Yao, Y. Yuan et al., "Targeting of tumour-infiltrating macrophages via CCL2/CCR2 signalling as a therapeutic strategy against hepatocellular carcinoma," Gut, vol. 66, no. 1, pp. 157-167, 2016.

[18] M. Cully, "Cancer: re-educating tumour-associated macrophages with nanoparticles," Nature Reviews. Drug Discovery, vol. 17, no. 7, p. 468, 2018.

[19] S. R. Gordon, R. L. Maute, B. W. Dulken et al., "PD-1 expression by tumour-associated macrophages inhibits phagocytosis and tumour immunity," Nature, vol. 545, no. 7655, pp. 495499, 2017.

[20] D. Pardoll, "Cancer and the immune system: basic concepts and targets for intervention," Seminars in Oncology, vol. 42, no. 4, pp. 523-538, 2015.

[21] L. C. de Erauso, M. Zuazo, H. Arasanz et al., "Resistance to PD-L1/PD-1 blockade immunotherapy. A tumor-intrinsic or tumor-extrinsic phenomenon?," Frontiers in Pharmacology, vol. 11, p. 441, 2020.

[22] G. Cerretelli, A. Ager, M. J. Arends, and I. M. Frayling, "Molecular pathology of Lynch syndrome," The Journal of Pathology, vol. 250, no. 5, pp. 518-531, 2020.

[23] I. M. Krzyzewska, S. M. Maas, P. Henneman et al., “A genomewide DNA methylation signature for SETD1B-related syndrome," Clinical Epigenetics, vol. 11, no. 1, p. 156, 2019. 
[24] I. Georgakopoulos-Soares, G. Koh, S. E. Momen, J. Jiricny, M. Hemberg, and S. Nik-Zainal, "Transcription-coupled repair and mismatch repair contribute towards preserving genome integrity at mononucleotide repeat tracts," Nature Communications, vol. 11, no. 1, p. 1980, 2020.

[25] N. K. Porkka, A. Olkinuora, T. Kuopio et al., "Does breast carcinoma belong to the Lynch syndrome tumor spectrum? Somatic mutational profiles vs. ovarian and colorectal carcinomas," Oncotarget, vol. 11, no. 14, pp. 1244-1256, 2020.

[26] J. Tiffen, S. J. Gallagher, F. Filipp et al., "EZH2 cooperates with DNA methylation to downregulate key tumor suppressors and IFN gene signatures in melanoma," The Journal of Investigative Dermatology, 2020.

[27] M. Butler, L. Pongor, Y. T. Su et al., "MGMT status as a clinical biomarker in glioblastoma," Trends Cancer., vol. 6, no. 5, pp. 380-391, 2020.

[28] L. Bonanno, A. Pavan, M. V. Dieci et al., "The role of immune microenvironment in small-cell lung cancer: distribution of PD-L1 expression and prognostic role of FOXP3-positive tumour infiltrating lymphocytes," European Journal of Cancer, vol. 101, pp. 191-200, 2018.

[29] J. S. J. Lim and R. A. Soo, "Nivolumab in the treatment of metastatic squamous non-small cell lung cancer: a review of the evidence," Therapeutic Advances in Respiratory Disease, vol. 10, no. 5, pp. 444-454, 2016.

[30] F. X. Schaub, V. Dhankani, A. C. Berger et al., "Pan-cancer alterations of the MYC oncogene and its proximal network across the Cancer Genome Atlas," Cell Systems, vol. 6, no. 3, pp. 282-300.e2, 2018.

[31] ICGC/TCGA pan-cancer analysis of whole genomes consortium, "Pan-cancer analysis of whole genomes," Nature, vol. 578, no. 7793, pp. 82-93, 2020.

[32] C. Calabrese, PCAWG Transcriptome Core Group, N. R. Davidson et al., "Genomic basis for RNA alterations in cancer," Nature, vol. 578, no. 7793, pp. 129-136, 2020.

[33] B. Rodriguez-Martin, PCAWG Structural Variation Working Group, E. G. Alvarez et al., "Pan-cancer analysis of whole genomes identifies driver rearrangements promoted by LINE-1 retrotransposition," Nature Genetics, vol. 52, no. 3, pp. 306-319, 2020.

[34] E. Rheinbay, P. C. A. W. G. Drivers, Functional Interpretation Working Group et al., "Analyses of non-coding somatic drivers in 2,658 cancer whole genomes," Nature, vol. 578, no. 7793, pp. 102-111, 2020.

[35] M. H. Bailey, C. Tokheim, E. Porta-Pardo et al., "Comprehensive characterization of cancer driver genes and mutations," Cell, vol. 173, no. 2, pp. 371-385.e18, 2018.

[36] Q. Liu, Y. Gao, and X. Ci, "Role of Nrf2 and its activators in respiratory diseases," Oxidative Medicine and Cellular Longevity, vol. 2019, 17 pages, 2019.

[37] M. de Freitas Silva, L. Pruccoli, F. Morroni et al., "The Keap1/Nrf2-ARE pathway as a pharmacological target for chalcones," Molecules, vol. 23, no. 7, p. 1803, 2018.

[38] V. Krajka-Kuźniak, J. Paluszczak, and W. Baer-Dubowska, "The Nrf2-ARE signaling pathway: an update on its regulation and possible role in cancer prevention and treatment," Pharmacological Reports, vol. 69, no. 3, pp. 393-402, 2017.

[39] P. Shaw and A. Chattopadhyay, "Nrf2-ARE signaling in cellular protection: mechanism of action and the regulatory mechanisms," Journal of Cellular Physiology, vol. 235, no. 4, pp. 3119-3130, 2019.
[40] E. Kubo, B. Chhunchha, P. Singh, H. Sasaki, and D. P. Singh, "Sulforaphane reactivates cellular antioxidant defense by inducing Nrf2/ARE/Prdx6 activity during aging and oxidative stress," Scientific Reports, vol. 7, no. 1, article 14130, 2017.

[41] A. L. Furfaro, N. Traverso, C. Domenicotti et al., "The Nrf2/HO-1 axis in cancer cell growth and chemoresistance," Oxidative Medicine and Cellular Longevity, vol. 2016, Article ID 1958174, 14 pages, 2016.

[42] J. Haapasalo, K. Nordfors, K. J. Granberg et al., "NRF2, DJ1 and SNRX1 and their prognostic impact in astrocytic gliomas," Histol Histopathology, vol. 33, no. 8, pp. 791-801, 2018.

[43] T. Ohnuma, K. Sakamoto, A. Shinoda et al., "Procyanidins from Cinnamomi cortex promote proteasome-independent degradation of nuclear Nrf2 through phosphorylation of insulin-like growth factor-1 receptor in A549 cells," Archives of Biochemistry and Biophysics, vol. 635, pp. 66-73, 2017.

[44] C. R. Boland and A. Goel, "Microsatellite instability in colorectal cancer," Gastroenterology, vol. 138, no. 6, pp. 2073-2087.e3, 2010.

[45] T. Armaghany, J. D. Wilson, Q. Chu, and G. Mills, "Genetic alterations in colorectal cancer," Gastrointest Cancer Research, vol. 5, no. 1, 2012.

[46] K. A. Szigeti, O. Galamb, A. Kalmár et al., "Role and alterations of DNA methylation during the aging and cancer," Orvosi Hetilap, vol. 159, no. 1, pp. 3-15, 2018.

[47] Y. Yin, K. Che, J. Hu et al., "Hypermethylation of the RSK4 promoter associated with BRAF V600E promotes papillary thyroid carcinoma," International Journal of Oncology, vol. 56, no. 5, pp. 1284-1293, 2020.

[48] M. Manoochehri, Y. Wu, N. A. Giese et al., "SST gene hypermethylation acts as a pan-cancer marker for pancreatic ductal adenocarcinoma and multiple other tumors: toward its use for blood-based diagnosis," Molecular Oncology, vol. 14, no. 6, pp. 1252-1267, 2020.

[49] M. R. Junttila and F. J. de Sauvage, "Influence of tumour micro-environment heterogeneity on therapeutic response," Nature, vol. 501, no. 7467, pp. 346-354, 2013.

[50] Z. Wang, K. Song, W. Zhao, and Z. Zhao, "Dendritic cells in tumor microenvironment promoted the neuropathic pain via paracrine inflammatory and growth factors," Bioengineered, vol. 11, no. 1, pp. 661-678, 2020.

[51] J. Berntsson, B. Nodin, J. Eberhard, P. Micke, and K. Jirström, "Prognostic impact of tumour-infiltrating B cells and plasma cells in colorectal cancer," International Journal of Cancer, vol. 139, no. 5, pp. 1129-1139, 2016.

[52] Y. Zhang, G. Yu, H. Chu et al., "Macrophage-associated PGK1 phosphorylation promotes aerobic glycolysis and tumorigenesis," Molecular Cell, vol. 71, no. 2, pp. 201-215.e7, 2018.

[53] T. Lianyuan, X. Dianrong, Y. Chunhui, M. Zhaolai, and J. Bin, "The predictive value and role of stromal tumorinfiltrating lymphocytes in pancreatic ductal adenocarcinoma (PDAC)," Cancer Biology \& Therapy, vol. 19, no. 4, pp. 296-305, 2018.

[54] M. G. Morvan and L. L. Lanier, "NK cells and cancer: you can teach innate cells new tricks," Nature Reviews. Cancer, vol. 16, no. 1, pp. 7-19, 2016.

[55] P. Sharma, S. Hu-Lieskovan, J. A. Wargo, and A. Ribas, "Primary, adaptive, and acquired resistance to cancer immunotherapy," Cell, vol. 168, no. 4, pp. 707-723, 2017.

[56] G. P. Dunn, A. T. Bruce, H. Ikeda, L. J. Old, and R. D. Schreiber, "Cancer immunoediting: from immunosurveillance to 
tumor escape," Nature Immunology, vol. 3, no. 11, pp. 991998, 2002.

[57] Z. Wang, G. Li, S. Dou et al., "Tim-3 promotes listeria monocytogenes immune evasion by suppressing major histocompatibility complex class I," The Journal of Infectious Diseases, vol. 221, pp. 830-840, 2019.

[58] F. Tang, Y. Xu, and B. Zhao, "NLRC5: new cancer buster?," Molecular Biology Reports, vol. 47, no. 3, pp. 2265-2277, 2020.

[59] H. Ohtani, "Focus on TILs: prognostic significance of tumor infiltrating lymphocytes in human colorectal cancer," Cancer Immunity, vol. 7, p. 4, 2007.

[60] S. Hadrup, M. Donia, and P. thor Straten, "Effector $\mathrm{CD}_{4}$ and CD8 T cells and their role in the tumor microenvironment," Cancer Microenvironment, vol. 6, no. 2, pp. 123-133, 2013.

[61] E. M. Bekes, B. Schweighofer, T. A. Kupriyanova et al., "Tumor-recruited neutrophils and neutrophil TIMP-free MMP-9 regulate coordinately the levels of tumor angiogenesis and efficiency of malignant cell intravasation," The American Journal of Pathology, vol. 179, no. 3, pp. 1455-1470, 2011.

[62] X. Shan, C. Zhang, Z. Wang et al., "Prognostic value of a ninegene signature in glioma patients based on tumor-associated macrophages expression profiling," Clinical Immunology, vol. 216, article 108430, 2020.

[63] J. Galon, H. K. Angell, D. Bedognetti, and F. M. Marincola, "The continuum of cancer immunosurveillance: prognostic, predictive, and mechanistic signatures," Immunity, vol. 39, no. 1, pp. 11-26, 2013.

[64] K.-W. Bi, X.-G. Wei, X.-X. Qin, and B. Li, "BTK has potential to be a prognostic factor for lung adenocarcinoma and an Indicator for tumor microenvironment remodeling: a study based on TCGA data mining," Frontiers in Oncology, vol. 10, p. 424, 2020. 